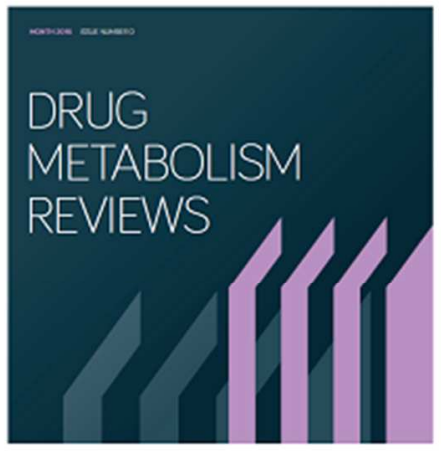

\title{
Metabolic disposition and biological significance of simple phenols of dietary origin: hydroxytyrosol and tyrosol.
}

\begin{tabular}{|c|c|}
\hline Journal: & Drug Metabolism Reviews \\
\hline Manuscript ID & LDMR-2016-0010 \\
\hline Manuscript Type: & Review \\
\hline Date Submitted by the Author: & 17-Mar-2016 \\
\hline Complete List of Authors: & 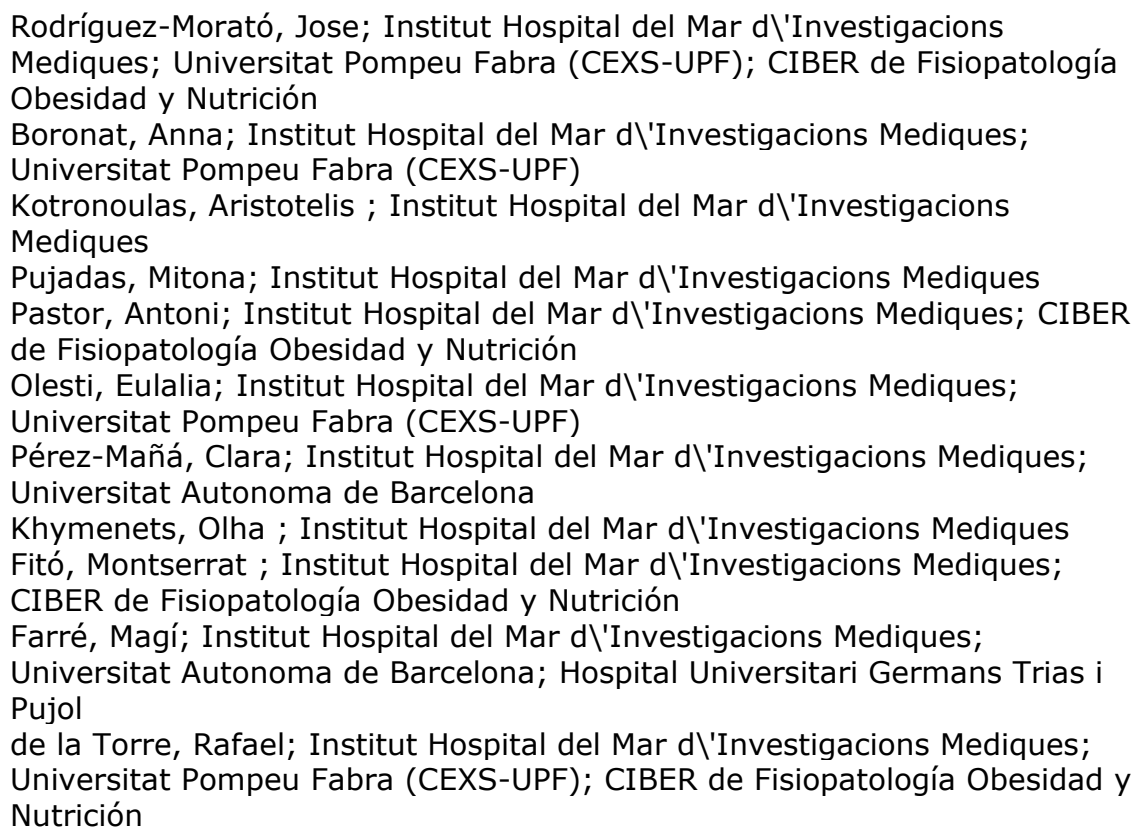 \\
\hline Keywords: & $\begin{array}{l}\text { Hydroxytyrosol, Tyrosol, Virgin olive oil, Red wine, Ethanol, Mediterranean } \\
\text { Diet, Cardiovascular prevention, Phenolic compounds, Dopamine, Tyramine }\end{array}$ \\
\hline
\end{tabular}




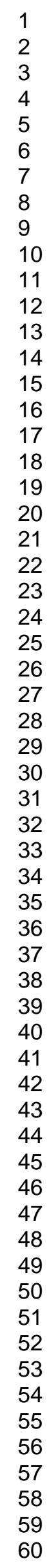

SCHOLARONE ${ }^{m}$ Manuscripts 10 11 13 14 15 16 17

21

23

24

26

27

29

30

32

33

34

35

36

39

40

41

42

44

45

46

47

48

49

50

52

53

54

56

57

58

60

URL: http:/mc.manuscriptcentral.com/ldmr 


\section{Metabolic disposition and biological significance of simple phenols of dietary origin: hydroxytyrosol and tyrosol.}

Jose Rodríguez-Moratón ${ }^{a, b, c}$, Anna Boronat ${ }^{a, b}$, Aristotelis Kotronoulas ${ }^{a}$, Mitona Pujadas ${ }^{a}$, Antoni Pastor $^{a, c}$, Eulalia Olesti ${ }^{a, b}$, Clara Pérez-Mañãa, ${ }^{a, d}$ Olha Khymenets ${ }^{a}$, Montserrat Fitóc,e, Magí Farréa, ${ }^{\mathrm{a}, \mathrm{f}}$, Rafael de la Torre ${ }^{a, b, c, *}$

${ }^{a}$ Integrative Pharmacology and Systems Neuroscience, IMIM (Hospital del Mar Research Institute), Dr. Aiguader 88, 08003, Barcelona, Spain. jrodriguez1@imim.es; aboronat@imim.es;

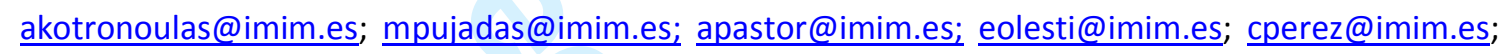
okhymenets@gmail.com; rtorre@imim.es

${ }^{\mathrm{b}}$ Universitat Pompeu Fabra (CEXS-UPF), Dr. Aiguader 88, 08003, Barcelona, Spain.

${ }^{c}$ CIBER de Fisiopatología Obesidad y Nutrición, Santiago de Compostela, 15706, Spain.

d Universitat Autònoma de Barcelona, Bellaterra, Spain.

${ }^{\mathrm{e}}$ Cardiovascular Risk and Nutrition Research Group, IMIM (Hospital del Mar Research Institute), Dr. Aiguader 88, 08003, Barcelona, Spain. mfito@imim.es

${ }^{\mathrm{f}}$ Hospital Universitari Germans Trias i Pujol (IGTP), Badalona, Spain. Mfarre.germanstrias@gencat.cat

*Corresponding author: Rafael de la Torre, PharmD, PhD. Integrative Pharmacology and Systems Neuroscience Research Group, IMIM (Hospital del Mar Medical Research Institute), Barcelona, Spain. Tel: +34 933160484; Fax: +34 933160467; E-mail:rtorre@imim.es

Keywords: Hydroxytyrosol, tyrosol, virgin olive oil, red wine, ethanol, Mediterranean Diet, cardiovascular prevention, phenolic compounds, dopamine, tyramine. 


\section{Abstract}

Hydroxytyrosol and tyrosol are dietary phenolic compounds present in virgin olive oil and wine. Both compounds are also endogenously synthesized in our body as byproducts of dopamine and tyramine metabolisms, respectively. Over the last decades, research into hydroxytyrosol and tyrosol has experienced an increasing interest due to the role that these compounds may play in the prevention of certain pathologies (e.g. cardiovascular, metabolic, neurodegenerative diseases, and cancer). The translation of promising in vitro and in vivo biological effects from preclinical studies to the context of human disease prevention initially depends on whether the dose ingested becomes available at the site of action. In this regard, information regarding the bioavailability and metabolic disposition of hydroxytyrosol and tyrosol is of most importance to evaluate the impact they may have on human health. In this review, we discuss and summarize the state of the art of the scientific evidence regarding the processes of absorption, distribution, metabolism, and excretion of both hydroxytyrosol and tyrosol. We also examine the impact of these compounds and their metabolites on biological activity in terms of beneficial health effects. Finally, we evaluate the different analytical approaches that have been developed to measure the plasma and urinary levels of hydroxytyrosol, tyrosol and their metabolites. 


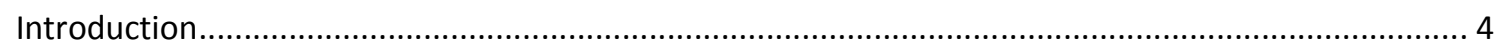

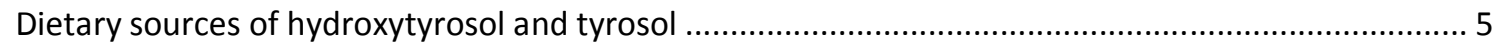

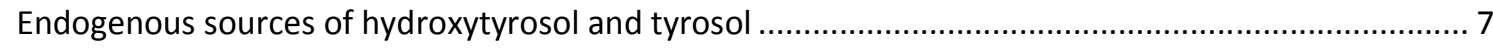

Endogenous formation of HT: Dopamine oxidative metabolism and its interaction with ethanol.... 7

Endogenous formation of Tyr: Tyramine oxidative metabolism and its interaction with ethanol..... 8

Human studies on endogenous formation of hydroxytyrosol and its interaction with ethanol intake 9

Human studies on endogenous formation of tyrosol and its interaction with ethanol intake ........ 11

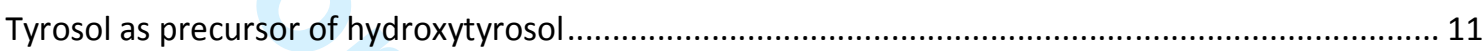

Dual effect of ethanol on hydroxytyrosol and tyrosol levels ................................................... 12

Metabolic disposition of hydroxytyrosol and tyrosol ............................................................. 13

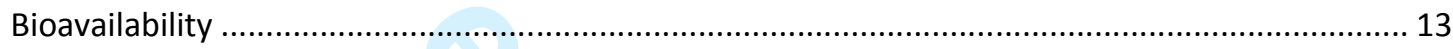

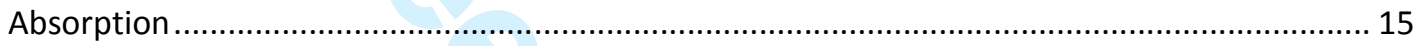

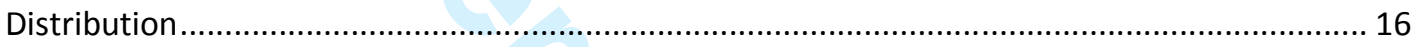

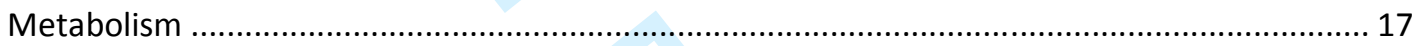

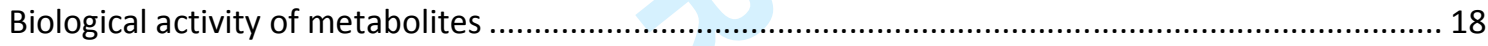

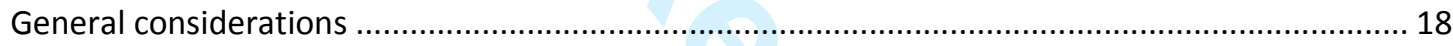

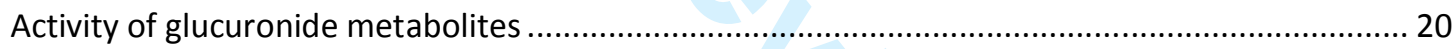

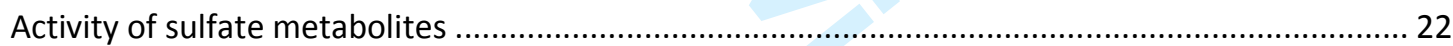

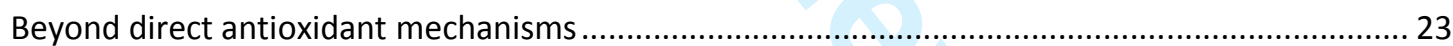

Analysis of hydroxytyrosol, tyrosol and their metabolites in biological matrices ............................. 24

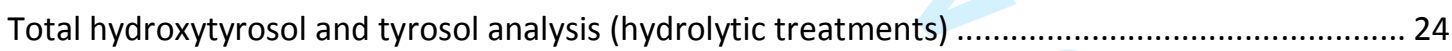

Hydroxytyrosol, tyrosol and main metabolite analysis (non-hydrolytic treatments) ....................25

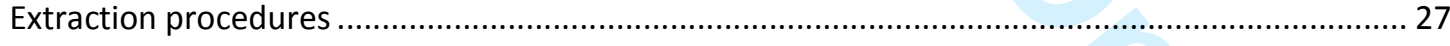

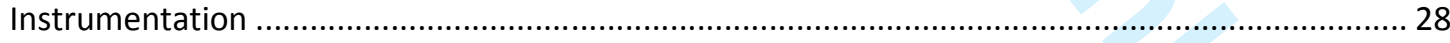

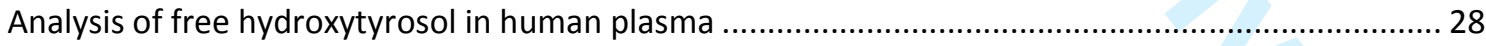

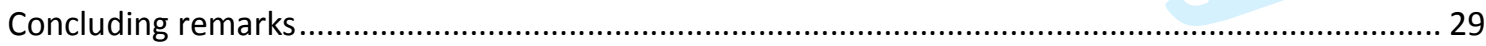




\section{Introduction}

Hydroxytyrosol [2-(3,4-dihydroxyphenyl)ethanol, HT] and tyrosol [2-(4hydroxyphenyl)ethanol, Tyr] (Figure 1) are simple phenolic compounds of natural origin that are also synthesized in our body. In nature, they play a defensive role against pathogens and herbivores (Ortega-García and Peragón, 2010). The main dietary sources of HT and Tyr are olive oil (Bayram et al., 2013; Servili et al., 2014) and wine (Piñeiro et al., 2011), while Tyr can be additionally found in other alcoholic beverages such as beer (Almeida et al., 2006) and vermouth (Chen et al., 2014).

As phenolic compounds HT and Tyr are expected to have strong antioxidant activities (Carrasco-Pancorbo et al., 2005) due to the potent redox properties of the phenolic hydroxyl groups and the structural relationships in the chemical configuration of the molecules (Cheng et al., 2002). Their biological activities in vivo, however, are mediated by mechanisms other than scavenging free radicals (Forman et al., 2014).

Extra-virgin olive oil, the main dietary source of $\mathrm{HT}$, has been shown to be cardioprotective in humans by: (1) increasing HDL-cholesterol; (2) reducing oxidative damage to lipids; (3) decreasing inflammation; (4) improving endothelial function; and (5) decreasing systolic blood pressure (Covas et al., 2015). The Mediterranean Diet, including extra-virgin olive oil, has been reported to prevent primary cardiovascular events (Estruch et al., 2013) and should be recommended to subjects at high risk for cardiovascular disease (Chiva-Blanch et al., 2014). In addition, moderate alcohol consumption, especially beverages rich in polyphenols such as wine, seems to confer cardiovascular protective effects not only in individuals at risk of cardiovascular disease, but also in healthy subjects (Chiva-Blanch et al., 2013).

In both olive oil and wine there has been debate concerning which components of these foods should be credited with health benefits. While some authors consider that the benefits are provided essentially by the fatty acid composition of olive oil (particularly oleic acid) and ethanol as a principal active ingredient in wine (Sharp, 2003; Vissers et al., 2004), others believe that the 
contribution of polyphenols is essential. A turning point in this controversy was the EUROLIVE clinical trial. It showed for the first time the importance of olive oil polyphenols with respect to health effects, particularly in protecting LDL oxidation, a hallmark in atherosclerosis progression (Covas et al., 2006b). On the basis of this clinical study, in November 2011, the European Food Safety Authority (EFSA) released a claim concerning the benefits of the daily ingestion of an olive oil rich in phenolic compounds, such as virgin olive oil. The EFSA Panel considered that, in order to bear the claim, $5 \mathrm{mg}$ of HT and its derivatives (e.g. oleuropein and Tyr) in olive oil should be consumed daily. These quantities, if provided by moderate amounts of olive oil, can be easily reached within the context of a balanced diet (EFSA, 2011). The final conclusion has been that all the components (fatty acids, ethanol, and phenolic compounds) are essential to understand the health benefits provided by these foods.

HT and Tyr have been associated with other health claims concerning beneficial effects in metabolic syndrome, cancer, and neurodegenerative diseases, although well designed and powered clinical trials are required to substantiate them (Granados-Principal et al., 2010; Visioli and Bernardini, 2011; Bernini et al., 2013; Bulotta et al., 2014; Rodríguez-Morató et al., 2015).

It is noteworthy that the effect of any dietary compound is influenced by the active bioavailable dose rather than the ingested one (Holst and Williamson, 2008). The present review will focus on the bioavailability of HT and Tyr with a particular emphasis on their metabolic disposition.

\section{Dietary sources of hydroxytyrosol and tyrosol}

As stated earlier, olive oil and wine are the major dietary sources of HT and Tyr. In the case of olive oil, its major components are fatty acids, of which the monounsaturated oleic acid represents from 55 to $83 \%$ of the total fatty acids, polyunsaturated fatty acids from 4 to $20 \%$, and saturated fatty acids from 8 to $14 \%$. The minor components constitute from 1 to $2 \%$ of the total content of an olive oil. They are grouped into two types: (1) the unsaponifiable fraction which can be extracted with solvents after the saponification of the olive oil and contains squalene and other triterpenes, 
sterols, tocopherols, and pigments; and (2) the soluble fraction which includes the phenolic acids, phenolic alcohols, flavonoids, and the glycosides, secoiridoids and the hydrolytic derivatives, anthocyanins, and hydroxycinnamic acid derivatives, which are the most studied and best-known components in terms of their health benefits (Covas et al., 2006c).

Olea europaea is rich in several phenol-conjugated oleosidic secoiridoids and oleosides present in olives and olive oil. Both HT and Tyr exist in the form of glycosides (known as HT-4- $\beta$-Dglucoside and salidroside) and secoiridoids. Secoiridoids are characterized by the presence of elenolic acid (in its glycosidic or aglyconic form) linked to HT and Tyr. In the olive, secoiridoids are generally bound to glucose (known as oleuropein and ligstroside), during the process of crushing and malaxation, however, $\beta$-glucosidases catalyze the deglycosylation that gives rise to their corresponding aglycones (Bendini et al., 2007). Oleuropein and ligstroside aglycones can be further hydrolyzed in the gut thus producing elenolic acid and the simple phenols HT and Tyr (Obied et al., 2008). The esters of HT and Tyr with deacetoxy elenolic acid are also biologically active compounds known as oleacin and oleocanthal, respectively (Karkoula et al., 2012)(Figure 2).

A number of factors including variety, olive fruit maturity, olive oil processing, and even agronomic factors determine the final amount of phenolic compounds in virgin olive oil, typically ranging between 100 and $600 \mathrm{mg} / \mathrm{kg}$. About half of this amount corresponds to $\mathrm{HT}$ and its derivatives (Tripoli et al., 2005).

Wine is another source of HT in our diet (Di Tommaso et al., 1998; Fernández-Mar et al., 2012). Tyr and HT could be considered as secondary metabolites from the tyrosine formed by yeasts during alcoholic fermentation. Furthermore, the synthesis of Tyr has been described as being directly proportional to the quantity of amino acids present in the must (Bordiga et al., 2016). Concentrations described in red wines (the mean of 30 Spanish wines) are $1 \mathrm{mg} / \mathrm{L}(0-5 \mathrm{mg} / \mathrm{L}$ ) for HT and $31 \mathrm{mg} / \mathrm{L}$ (20$40 \mathrm{mg} / \mathrm{L}$ ) for Tyr (Piñeiro et al., 2011). 
In addition to these dietary sources, HT can be found in plants in the following secoiridoids: (1) verbascoside (a caffeoyl phenylethanoid glycoside that is an ester formed with the phenylethanoid HT, the phenylpropanoid caffeic acid and the sugar alpha-L-rhamnopyranosyl-( $1 \rightarrow 3)$ ß-D-glucopyranose), (2) echinacoside (a caffeic acid glycoside from the phenylpropanoid class, constituted from a trisaccharide consisting of two glucose and one rhamnose moiety glycosidically linked to one caffeic acid and one HT residue at the centrally situated rhamnose), and (3) in several forsythosides (caffeoyl phenylethanoid glycosides found in extracts of Forsythia suspensa, a medicinal herb well belonging to the Oleacea family).

\section{Endogenous sources of hydroxytyrosol and tyrosol}

Endogenous formation of HT: Dopamine oxidative metabolism and its interaction with ethanol

Dopamine is initially metabolized by monoaminooxidase B (MAO-B) which gives rise to the aldehyde metabolite 3,4-dihydroxyphenylacetaldehyde (DOPAL). DOPAL is further oxidized by mitochondrial aldehyde dehydrogenase (ALDH) to the acid metabolite 3,4-dihydroxyphenylacetic acid (DOPAC), which is the major metabolite of dopamine in biological matrices (oxidative pathway). A minor metabolic pathway in dopamine metabolism is the reduction of DOPAL by aldose/aldehyde reductases to 3,4-dihydroxyphenylethanol (DOPET), also known as HT (Hashimoto et al., 2004) (Figure 3). DOPET is, therefore, simultaneously a dopamine metabolite and a dietary phenol with antioxidant properties. MOPET (4-hydroxy-3-methoxyphenylethanol or HVAlc) is the methylated metabolite of HT (also present in olive oil), while homovanillic acid (HVA) is the main metabolite of DOPAC. HT and HVAlc are present physiologically in low concentrations in biological matrices while DOPAC and HVA are more abundant, and the latter is a typical biomarker of dopamine turnover in clinical chemistry (Figure 3). 
On the other hand, ethanol ingested at moderate doses is metabolized to acetaldehyde by hepatic oxidative metabolism in a reaction regulated by the low $K_{m}$ alcohol dehydrogenase (ADH). In turn, acetaldehyde is converted to acetic acid (acetate) by acetaldehyde dehydrogenase (ALDH). Both oxidation reactions are responsible for reduced nicotinamide adenine dinucleotide production (NADH) (Vasdev et al., 2006).

Studies in animals showed an increase of DOPET formation due to the presence of ethanol (Davis and Walsh, 1970; Davis et al., 1970). Furthermore, in another study with rat liver slices (Tank and Weiner, 1979), the addition of ethanol changed the ratio DOPAC/DOPET from 10 to 0.25 , suggesting a shift in dopamine oxidative metabolism. The reductive environment created during alcohol metabolism was thought to be responsible for the change in the aldehyde (DOPAL) metabolism enhancing the formation of the alcohol derivative (DOPET or HT) instead of the acid one (DOPAC) (Tank and Weiner, 1979). A shift similar to that previously described was reported for serotonin, where the alcohol metabolite 5-hydroxytryptophol was preferably produced after ethanol intake instead of the 5-hydroxyindolacetic acid (Davis et al., 1967).

\section{Endogenous formation of Tyr: Tyramine oxidative metabolism and its interaction with ethanol}

Tyramine (4-hydroxyphenylethylamine) is a monoamine compound derived from the decarboxylation of the amino acid tyrosine through tyrosine decarboxylase. In a similar way to other biogenic amines, tyramine is deaminated by MAO forming an aldehyde intermediate which can be either oxidized by aldehyde dehydrogenase (generating 4-hydroxyphenylacetic acid) or reduced by alcohol dehydrogenase (generating tyrosol) (Figure 4) (Panova et al., 1997).

Tyr formation from tyramine after ethanol administration was initially proposed by Tacker in rats (Tacker et al., 1970). The animals were administered intraperitoneally with ethanol $(2 \mathrm{~g} / \mathrm{kg})$ followed by a labelled tyramine (tyramine- $-1{ }^{14} \mathrm{C}$ ) dose. The control group received intraperitoneal tyramine but no ethanol. Urine was collected for up to $96 \mathrm{~h}$ following administration for metabolite quantification, and results showed a 20 -fold increase in tyrosol $-{ }^{14} \mathrm{C}$ after ethanol administration. In a 
similar way to that which occurs in dopamine metabolism, ethanol increases the subsequent reduction of the previous aldehyde to the corresponding alcohol (4-hydroxyphenylethanol or Tyr) and decreases its oxidation to the corresponding acid (4-hydroxyphenylacetic acid or 4-HPAA). An increase in $\mathrm{NADH} / \mathrm{NAD}$ ratio during ethanol metabolism, and a competitive inhibition of ALDH by acetaldehyde, have been proposed as possible mechanisms for the observed shift in tyramine oxidative metabolism (Tacker et al., 1970).

\section{Human studies on the endogenous formation of hydroxytyrosol and its interaction with ethanol intake}

Within the context of bioavailability studies concerning phenolic compounds from wine and grape products, the urinary pharmacokinetics of HT in volunteers that had ingested red wine was compared to that after virgin olive oil ingestion. It was found that despite the 5 times higher dose of HT in olive oil (1.7 mg for olive oil/in $25 \mathrm{~mL}$ vs. $0.35 \mathrm{mg}$ for red wine/in $150 \mathrm{~mL}$ ) urinary recoveries of HT were higher after red wine administration. Moreover, these recoveries could not be explained by the dose of administered red wine, as they were $200 \%$ times greater than expected. At the time, it was hypothesized that an interaction between ethanol and dopamine metabolism could explain the increase in HT levels (de la Torre R, 2006).

Furthermore, in an intervention trial intended to demonstrate the effects of a Mediterranean-style diet on the primary prevention of cardiovascular disease, it was observed that baseline HT urinary concentrations correlated not only with wine intake, but also with self-reported ethanol consumption. This observation was obtained in a subsample of 1009 subjects from the PREDIMED study (Schröder et al., 2009).

In vivo evidence of HT endogenous formation after ethanol intake in humans was obtained in a double-blind, randomized, crossover, and controlled clinical trial designed to establish the 
contribution of the dose of ethanol on HT formation (DOPET-1 study). 24 healthy male volunteers were distributed in three different cohorts and each one received two doses of ethanol or placebo. Six different doses of ethanol $(6,12,18,24,30$, and $42 \mathrm{~g})$ were tested and urinary excretion of HT (from 0 to 6 hours after administration) was measured. HT excretion increased in relation to the administered dose of ethanol. On average, each gram of ethanol augmented the log-HT by 0.026 units (95\%-Cl: $[0.02,0.04] ; \mathrm{p}<0.001)$. HT was excreted in urine mainly as sulfate. The excretion of the sulfated and the methylated (HVAlc) metabolites of HT also rose with the ethanol administered dose, although this relationship was not found with HT glucuronides. The amount of free HT excreted in urine was very low and apparently unrelated to ethanol dose. Moreover, in the same study, a reduction in the ratio DOPAC/DOPET from placebo to the highest dose (42 $\mathrm{g}$ of ethanol) was observed (from 14 to 3.6 ), compatible with the occurrence of the shift in the dopamine oxidative metabolism to preferentially produce HT instead of DOPAC (Pérez-Mañá et al., 2015a).

A second study (Pérez-Mañá et al., 2015b) (DOPET-2) was conducted to assess the capacity of wine, wine polyphenols, and alcohol to promote endogenous HT generation in humans. It was a crossover, double-blind, randomized, and controlled clinical trial where red wine and vodka (15 g of ethanol dose in both cases), dealcoholized red wine (DW), and placebo (water) were administered to 28 healthy male volunteers. Urinary HT excretion was measured for a period of 0 to $6 \mathrm{~h}$ after administration. Results showed that HT recovery after wine and DW was $420 \%$ and $161 \%$ the amount ingested, respectively, indicating HT endogenous generation also after wine intake. The highest HT excretion was observed after wine in comparison with the other beverages. In turn, excretion after DW was higher than vodka and placebo, and that of vodka higher than placebo. As in the previous study with ethanol (Pérez-Mañá et al., 2015a), HT was mainly excreted as sulfate after all the beverages, while glucuronide and free form excretion was minor. HVA excretion was higher in ethanol-free wine in comparison with wine, and the same happened for DOPAC although the difference did not reach statistical significance. Additionally, the DOPAC/DOPET ratios after vodka and wine administration, were 2.5 -fold and 12.7 -fold lesser, respectively, than after placebo. The 
results of this second trial were also compatible with the DOPET promoting formation of the ethanol containing beverages.

\section{Human studies on endogenous formation of Tyr and its interaction with ethanol intake}

In humans, the first experimental evidence of Tyr formation after ethanol intake was obtained in the DOPET 1 study where Tyr excretion was measured as a secondary outcome (PérezMañá et al., 2015a). Tyr urinary excretion in the collection period from 0 to 6 hours after ethanol administration (from 6 to $42 \mathrm{~g}$ ) rose with the ethanol administered dose. On average, each gram of ethanol increased the log-Tyr by 0.051 units (95\%-Cl: [0.04, 0.07]; $p<0.001)$.

In the second clinical trial (DOPET 2) conducted in healthy volunteers (Pérez-Mañá et al., 2015b) total Tyr excretion after vodka was around 2-fold greater than after placebo. On the other hand, 4-HPAA excretion with DW was significantly higher than with wine while no differences were found between vodka and placebo. The ratio 4-HPAA/Tyr was higher in DW in comparison with wine, and the same happened for placebo in comparison with vodka. Results obtained were compatible with the presence of a shift in tyramine metabolism from an oxidative to a reductive pathway due to the presence of ethanol in both beverages, thus enhancing Tyr formation versus that of 4-HPAA.

\section{Tyrosol as precursor of hydroxytyrosol}

Despite the well-established, ethanol-induced endogenous formation of HT via interaction with the dopamine oxidative metabolism, the amounts of this phenolic compound recovered in urine after wine intake were very high and could not only have been due to this mechanism. In order to study additional sources of HT, three different potential precursors of this compound were considered: tyramine, tyrosol, and tyrosine. These compounds were selected because they are present in wine and have a common 4-hydroxyphenylethyl moiety (Figure 5). The potential conversion of these compounds into HT was evaluated following the individual administration of each one in an animal model (rats, doses of 10 and $20 \mathrm{mg} / \mathrm{kg}$ ). Additionally, the effect of ethanol by 
itself, or combined with each compound, was also examined. Compounds were administered intraperitoneally to 96 rats after previous ethanol $(1 \mathrm{~g} / \mathrm{kg})$ or saline administration. Urine was collected from 0 to 4 hours after administration. The results of these experiments identified tyrosol as a precursor of HT due to the fact that Tyr administration promoted HT urinary excretion in a dosedependent manner. Neither tyrosine nor tyramine were found to generate HT (Pérez-Mañá et al., 2015b). Tyramine was shown to be a precursor of tyrosol formation as discussed previously. Neither tyrosol nor HT was reported after administering ethanol alone, although both compounds were observed if tyramine or tyrosol were combined with ethanol.

Recent studies using human liver microsomes have identified the involvement of cytochrome P450 (CYP) in the conversion of Tyr into HT. Additional studies employing selective enzymatic inhibitors and recombinant baculovirus expressing cDNA from human cytochrome P450 have identified CYP2A6 and CYP2D6 as the two primary isoenzymes catalyzing this reaction (RodríguezMorató(2016)FoodChem).

\section{Dual effect of ethanol on Tyr and HT levels}

As has been previously mentioned, small amounts of Tyr and HT are endogenously generated as byproducts of tyramine and dopamine metabolism. In this regard, ethanol by itself alters these metabolic pathways so that a higher production of reduced metabolites (Tyr and HT) is produced at the expense of the oxidized ones (4-hydroxyphenylacetic acid and DOPAC, respectively) (Figures 3 and 4).

When ethanol is administered with phenolic compounds it displays a dual role. On the one hand, it has been proposed that ethanol present in red wine could promote the absorption of flavonoids (Dragoni et al., 2006). In the case of Tyr, studies with rats show that the urinary recovery of this phenolic compound is enhanced following ethanol administration, a fact that is compatible with an increase in its bioavailability. Parallel studies in humans showed that Tyr and HT urinary 
excretion levels were higher after they ingested alcohol-containing wine than after the same alcoholfree wine (Pérez-Mañá et al., 2015b). Taken together, the previous data suggest that ethanol increases the bioavailability of these phenolic compounds (known to be very low) by facilitating their absorption.

On the other hand, in the particular case of the conversion of Tyr into HT, ethanol plays a moderate inhibitory effect. Indeed, studies in rats demonstrate that the recoveries of HT following Tyr administration are higher than those following the administration of Tyr and ethanol (PérezMañá et al., 2015b). In vitro studies with human liver microsomes also confirm that ethanol has an inhibitory effect on HT formation from Tyr, probably due to an inhibitory effect on CYP (RodríguezMorató(2016)FoodChem).

It is worth mentioning that, according to the data published on human intervention studies, the highest levels of urinary HT described so far were reached after the administration of wine with ethanol. The most probable major mechanisms are: (1) an increase of Tyr (and HT) bioavailability due to ethanol, (2) an endogenous CYP-catalyzed conversion of Tyr into HT (slightly inhibited by ethanol) and, to a lesser extent, (3) an ethanol-induced rise in both Tyr and HT production following an alteration in tyramine and dopamine oxidative metabolisms (Figure 6).

\section{Metabolic disposition of Hydroxytyrosol and Tyrosol}

\section{Bioavailability}

As stated earlier, the most abundant phenols in olive oil are the nonpolar oleuropein- and ligstroside-aglycones, their glycosylated secoiridoids, and the polar HT and Tyr. A preliminary question when examining the bioavailability of these compounds would be whether non-polar aglycones are bioavailable or merely precursors of HT and Tyr. There are two studies supporting the latter. In research performed with Caco-2 cell monolayers and rat segments of jejunum and ileum, $\mathrm{HT}$ and Tyr were absorbed and the object of several metabolic reactions at the intestinal epithelium 
level whilst oleuropein was rapidly degraded by the colonic microflora resulting in the formation of HT (Corona et al., 2006). In humans, in a very well-designed study comparing the bioavailability of oleuropein-glycoside (the secoiridoid) in ileostomy patients versus subjects with a colon, it was concluded that oleuropein was recovered mainly as HT (Vissers et al., 2002). Therefore, at dietary doses (although local biological effects of aglycones and secoiridoids in the intestinal tract should not be discarded), the secoiridoids are essentially biotransformed by gut microflora thus giving rise to the simple phenols HT and Tyr. When oleuropein was administered at non-dietary higher doses, a small fraction of this compound was detected in body fluids as oleuropein metabolites/derivatives (Serra et al., 2012; García-Villalba et al., 2014).

The gender differences and their impact on HT bioavailability is another subject that requires further consideration. Until the last decade of the 20th century, research on women was neglected and results obtained in men were directly translated to women, both in medicine and in nutrition. In particular, sex-gender differences appear in xenokinetics and xenodynamics of phenolic compounds. Nonetheless, there is a lack of adequate sex-related studies (Campesi et al., 2014). Konstantinidou et al. have reported gender differences in gene expression changes in human peripheral blood mononuclear cells after virgin olive oil consumption, despite the low number of participants (Konstantinidou et al., 2010). A study with olive leaf extracts administration in humans noted a large interindividual variation in absorption and metabolism of phenolic compounds, possibly resulting from differences in human enzymatic activity (De Bock et al., 2013a). Males may be more efficient at conjugating oleuropein, which would explain their lower AUC for oleuropein but higher AUC for HT metabolites (De Bock et al., 2013b).

Regarding all this information we have reviewed a PREDIMED study subsample (Estruch et al., 2006) where total HT concentrations were measured in urine after a traditional Mediterranean diet. Ethanol consumption has been taken into account by analyzing ethyl glucuronide as a biomarker of alcohol usage. This normalization has been performed due to the fact that ethanol promotes HT 
endogenous generation in humans (Pérez-Mañá et al., 2015a) and significant differences in ethanol consumption exist between men and women (Schröder et al., 2009). An analysis of covariance (ANCOVA) was performed of total HT urine concentration of 341 male and 379 female adjusting for ethyl glucuronide as a continuous variable and gender as categorical variable (Figure 7). It was concluded that there were significant differences between gender $(P<0.001)$ confirming thus the gender dimorphism.

\section{Absorption}

Pioneering work by Manna et al using differentiated Caco-2 cell monolayers as a model system examined for the first time the mechanisms of HT absorption. They concluded that HT transport occurs via a bidirectional passive diffusion mechanism, and estimated that the molecule was quantitatively absorbed at the intestinal level (Manna et al., 2000). The first study on absorption in rats was from the group of Visioli et al. who demonstrated that HT was dose-dependently absorbed and excreted in urine mostly as a glucuronide conjugate (Visioli et al., 2001). Further research in humans confirmed the bioavailability of HT and Tyr after the administration of olive oil (Caruso et al., 2001; Miró-Casas et al., 2001b; Miró-Casas et al., 2003a; Miró-Casas et al., 2003b) also in a dose-dependent manner (Weinbrenner et al., 2004; Covas et al., 2006a). These preliminary studies showed that while absorption of HT and Tyr was reasonably good, (Vissers et al., 2002), bioavailability was extremely poor. This was due to an extensive first pass metabolism in the gut and liver as concentrations of these phenolic compounds in urine were extremely low (about 5 to 10\% recovered in their free forms, respectively) (Caruso et al., 2001), and in plasma the free forms of HT and Tyr were deemed undetectable (Miró-Casas et al., 2003a).

Studies in rat models also provided some additional clues on the bioavailability of HT and Tyr. In the case of $\mathrm{HT}, 24 \mathrm{~h}$ urinary recoveries using a tritiated analog demonstrated that while the dose recovery was almost complete when administered with an oil matrix $(94 \%)$ it was much lower in a 
water one (71\%). The same figures were observed for Tyr: $75 \%$ versus $53 \%$ for both matrices, respectively (Tuck et al., 2001). In a study comparing the bioavailability of HT in humans it was also observed that recoveries were much higher after its administration as a natural component of olive oil (44.2\% of HT administered) than after its addition to refined olive oil ( $23 \%$ of HT administered) or yogurt (5.8\% of dose or approximately $13 \%$ of that recorded after virgin olive oil intake) (Visioli et al., 2003). In non-dietary doses (2.5 mg/kg body weight) of purified HT (99.5\%) administered in an aqueous solution it has been estimated that bioavailability was $5 \%$ and absorption $9 \%$ (GonzálezSantiago et al., 2010a).

Such observations lead to the conclusion that HT and Tyr absorption whilst quite good is matrix-dependent. However, as explained below, bioavailability is poor due to an extensive metabolism. Figures on the rates of absorption and bioavailability may vary among authors depending on the analytical approach applied and the number of metabolites monitored (in turn very much dependent on the availability of reference standards). Globally, some authors question to what extent phenolic compounds such as HT and Tyr at dietary doses are responsible for any biological effect (Vissers et al., 2004). Concentrations reached of free phenolic compounds (the ones tested in vitro or ex vivo) are too low to provide any direct antioxidant effect. Nevertheless, the potential biological activity of metabolites and the nutrigenomic effects on biological systems are alternative explanations to our understanding of the health benefits associated with dietary exposure to HT and Tyr (Konstantinidou et al., 2013).

\section{Distribution}

Once absorbed, HT is widely distributed throughout the organism. Pioneering studies in this field were performed by D'Angelo et al., who administered an intravenous dose of $1.5 \mathrm{mg} / \mathrm{kg}$ of $\left[{ }^{14} \mathrm{C}\right]-$ labeled HT to rats. The pharmacokinetic analysis indicated an extensive and fast uptake of this antioxidant by different organs including skeletal muscle, kidneys, liver, lungs, heart, and brain 
(D'Angelo et al., 2001). The ability of HT to reach the brain after crossing the blood-brain barrier demonstrated in vivo that it fulfills this essential requirement to be used as a neuroprotective agent.

Recently, further evidence supporting HT distribution has been published. In a study in rats, the oral administration of increasing doses $(1,10$, and $100 \mathrm{mg} / \mathrm{kg}$ ) of this compound (given in a refined olive oil matrix), demonstrated that $\mathrm{HT}$ accumulated in a dose-dependent manner not only in urine and plasma, but also in the liver, kidney, and brain (López de las Hazas et al., 2015).

In the two previously mentioned studies in rats, the kidneys and the liver were the organs that presented higher uptakes of hydroxytyrosol and its metabolites.

\section{Metabolism}

The number of HT metabolites reported in the bibliography has increased during recent years. So far, more than 10 metabolites have been described. These include $O$-methylated forms (Caruso et al., 2001), aldehydes and acids formed via oxidation of the aliphatic alcohol (D'Angelo et al., 2001), sulfates (Tuck et al., 2002), glucuronides (Khymenets et al., 2011), and acetylated and sulfated derivatives (Rubió et al., 2012) as well as an $\mathrm{N}$-acetylcysteine derivative (Kotronoulas et al., 2013). In the case of the latter, it was identified in a recent study in rats in which the dose-dependent metabolic disposition of HT was investigated at three different doses $(1,10$, and $100 \mathrm{mg} / \mathrm{kg})$. In this study, the potential detection of HT mercapturates ( $\mathrm{N}$-acetyl-5-S-cysteinyl-HT) resulting from the autoxidation of HT and adduct formation with glutathione was evaluated in urine. Following HT administration, dose-dependent variations in recovery of all the metabolites evaluated were observed. At the lowest dose of $1 \mathrm{mg} / \mathrm{kg}$, the glucuronidation pathway was the most relevant (25$30 \%$ ), with lower recoveries for sulfation (14\%), while at the highest dose of $100 \mathrm{mg} / \mathrm{kg}$, sulfation was the most prevalent (75\%). The mercapturate conjugate of HT was formed in a dose-dependent manner (Kotronoulas et al., 2013). 
Regarding the enzymes involved in HT metabolism, we can differentiate between two major families. The enzymes implicated in HT phase I metabolism are non-microsomal alcohol and aldehyde dehydrogenases, both located in the cytosol. The corresponding metabolites are DOPAL and DOPAC which are non-specific as they are not only metabolites of dietary $\mathrm{HT}$, but also of dopamine. On the other hand, the enzymes involved in HT phase II reactions are sulfotransferases (SULT), uridine 5'-diphosphoglucuronosyl transferases (UGTs), catechol-O-methyltransferases (COMT), and acetyltransferases. A metabolite resulting from methylation and glucuronidation has also been identified, although this represents a minor metabolic pathway (Miró-Casas et al., 2003a; Khymenets et al., 2011). A general representation of HT metabolites is depicted in Figure 8.

\section{Biological activity of metabolites}

\section{General considerations}

To date, practically all the studies have been focused on assessing the biological activities of parent phenolic compounds, HT and Tyr and, as a result, a considerable amount of data has accumulated (for detailed information refer to (Visioli et al., 1998; Visioli et al., 2002; GranadosPrincipal et al., 2010; Gil-Izquierdo et al., 2014; Rodríguez-Morató et al., 2015)), but practically nothing is known about the activity of their principal metabolites. As previously stated, the concentrations of HT and Tyr themselves in biological fluids are extremely low compared to their metabolites (Miró-Casas et al., 2001b; Miró-Casas et al., 2003a; González-Santiago et al., 2010b; Kotronoulas et al., 2013; Pastor et al., 2016) . There is, therefore, good reason to take into consideration the participation of metabolites, along with their parent compounds, with respect to the beneficial effects reported for dietary consumed HT and Tyr.

In spite of the increasing knowledge about the metabolism of HT and Tyr, there is sparse data regarding the activity of their major metabolites, phase II conjugates. This is mainly due to the lack of a good description of their disposition and adequate reference compounds. Recently, the 
chemical/biological synthesis of some of the metabolites, including glucuronides (Khymenets et al., 2006; Lucas et al., 2009) and sulfates (Gomes et al., 2015), has been reported. The availability of these products (either through in-house or customized synthesis) has facilitated accessibility to standards. This fact has had a positive impact on HT and Tyr bioavailability and disposition research, which is reflected in the latest achievements and increased activity in this research field (Deiana et al., 2011; Giordano et al., 2015; Atzeri et al., 2016). Within this framework, the bioactivity of dietary HT and Tyr has been reevaluated with current, greater knowledge about their metabolite bioavailability and evaluation as bioactive compounds.

When HT research first commenced, the obstacle caused by the unavailability of standards was overcome by preparing the HPLC-extracts in urine metabolites within a metabolic study on the bioavailability of tritium labelled HT in rats (Tuck et al., 2002). The extracts were characterized and the structures of identified phase II metabolites of HT (monoglucuronides, monosulfates, and several methylated conjugates) were assessed using mass spectrometry and nuclear magnetic spectroscopy techniques (Tuck et al., 2002). Moreover, an indirect identification was performed using enzymespecific hydrolysis (Tuck et al., 2001). The authors compared the scavenging properties of the rat urine HPLC extracts of identified metabolites using the radical 2,2-diphenyl-1-picrylhydrazyl (DPPH test). They observed that the most potent scavenger was HT-3-O-glucuronide followed by parent HT and its methylated metabolites homovanillic acid (HVA) and homovanillyl alcohol (HVAlc), whereas the extract of HT monosulfate was almost devoid of activity.

Such conclusions had far reaching effects since at that time the glucuronide metabolites of HT were believed to be principal metabolites available within human body in response to HT intervention (Visioli et al., 2001; Covas et al., 2003; Miró-Casas et al., 2003a; Miró-Casas et al., 2003b), and the radical scavenging activities were considered to be essential to exhibit direct biological activity according to the free-radical theory of aging/age-related diseases (Harman, 1956; 
Rahman, 2007). Consequently, it was anticipated that the glucuronide metabolites would be responsible for a number of in vivo health benefits of dietary HT.

\section{Activity of glucuronide metabolites}

To corroborate the previous assumption, the antioxidant activities of HT glucuronides were reconsidered within a range of concentrations compatible with their dietary consumption (Khymenets et al., 2010). Firstly, the biocatalytic synthesis and purification of the monoglucuronide conjugates of HT, Tyr, and HVAlc using porcine liver microsomes was developed and scaled up to obtain analytically pure products in mg-range (Khymenets et al., 2006). Secondly, to estimate the biologically relevant range of glucuronide metabolite concentrations of $\mathrm{HT}\left(3^{\prime}-\mathrm{O}-\right.$ and $4^{\prime}-\mathrm{O}-$ glucuronides), Tyr, and HVAlc (both 4'-O-glucuronide) and their core compounds, a direct LC-MS/MS approach was developed. The synthesized and reference compounds were applied to human urine samples after an intervention study with a dietary dose of virgin olive oil (Khymenets et al., 2011). Finally, because free radical activities play a central role in atherogenic LDL oxidation (Witztum, 1994), phenolic compounds and their glucuronides were assessed for their radical-scavenging capacities using chemical DPPH assay (hydrogen donation abilities). In addition, they were evaluated for their abilities to inhibit in vitro Cu-mediated LDL oxidation at the concentration ranges observed in human biological fluids (range, 0.01-10 $\mu \mathrm{M}$ ) after dietary virgin olive oil consumption (Khymenets et al., 2010). In these assays, none of the glucuronides displayed significant antioxidant activities in both experiments at the tested concentrations in contrast to some of their parent compounds. To some extent HVAlc, and especially HT, were shown to be strong radical scavengers in agreement with earlier reports (Briante et al., 2003; Roche et al., 2005).

Such results were in line with a previous theoretical study in which phase II metabolites glucuronides (and also sulfates) were predicted to lose the antiradical activity characteristic of their parent compound (Nenadis et al., 2005). Subsequently, these mechanistic and in vitro tests showed that glucuronide metabolites do not contribute with direct antioxidant effects to the modulation that 
had been observed in human oxidative status (e.g. previously reported impacts on lipid and LDL oxidation (Fitó et al., 2005) or serum antioxidant capacity (Fitó et al., 2002)).

In contrast to the previously mentioned reports, another study (Deiana et al., 2011) reported that HT glucuronide metabolites, specifically the mix of $3^{\prime}-O-\beta-D-$ and $4^{\prime}-O-\beta-D-$ glucuronidated isoforms, were able to protect renal cells (LLC-PK1 cells as a culture model) against $\mathrm{H}_{2} \mathrm{O}_{2}$-induced lipid peroxidation-related membrane oxidative damage by reducing malondialdehyde production and modifying the profile of the major oxidizable membrane lipids, unsaturated fatty acids, and cholesterol. Nonetheless, glucuronide metabolites acted to lesser extent than parent HT. On the other hand, in marked contrast to $\mathrm{HT}$, its glucuronides were shown to have no effect on $\mathrm{H}_{2} \mathrm{O}_{2}$ induced cell death through their interaction with intracellular oxidative stress cell survival signaling pathways (phosphorylation state of the kinases ERK $1 / 2$ and Akt/PKB was evaluated). No release of free HT through either hydrolytic or enzymatic cleavage of the glucuronide metabolites both in the medium and in the cells was confirmed by specific tests. The authors suggested that increased polarity/hydrophilicity due to glucuroconjugation had limited the access of these phase II metabolites of HT to the cell (no detectable uptake of HT glucuronides in LLC-PK1 cells was reported). Therefore, in contrast to parent $\mathrm{HT}$, its phase II metabolites were unable to interact with intracellular signaling pathways. It was hypothesized that the protection of renal cells by glucuronide metabolites was probably achieved through a direct scavenging of initiating aqueous radicals which originated from the reaction with $\mathrm{H}_{2} \mathrm{O}_{2}$, both in the reaction medium and near the membrane surface. On the other hand, HT was thought to also potentially act at an intra-cellular level (Deiana et al., 2008; Deiana et al., 2011).

Based on these data, further experiments should be designed to reveal the mechanism of this scavenging, and thus confirm whether it is relevant for in vivo protection against $\mathrm{H}_{2} \mathrm{O}_{2}$-mediated pathological processes where the lipid peroxidation process plays a central role. 
In a different study, HT glucuronides, but not HVAlc glucuronide, were also shown to protect red blood cells (RBC) from in vitro $\mathrm{H}_{2} \mathrm{O}_{2}$-induced oxidative injury at low concentrations (Paiva-Martins et al., 2013). At higher concentrations, however, the effect of protection for HT glucuronides remained practically the same as at low doses. The authors postulated that some sort of saturation of glucuronide specific RBC transporters could have occurred, limiting the availability of glucuronides inside RBC, or/and the glucuronides may have undergone a restricted hydrolysis to liberate active HT, which could then be absorbed by the cells. However, no experimental data supported this hypothesis. Recently, it has been shown that RBC can play a significant role in the bioavailability of circulating HT metabolites, and that in situ deconjugation of HT metabolites into free form could take place in rat RBC according to the intracellular pharmacokinetics of detected metabolites and free HT. The authors, therefore, suggested that there could be a mechanism of protection against cell oxidative damage in RBCs by HT metabolites (Rubió et al., 2014).

Based on the accumulative data with respect to the correlation between HT consumption and in vivo antioxidant/anti-inflammatory effects (Bogani et al., 2007), indirect mechanisms involving HT main metabolites in this relationship were investigated. Among them, tissue/organ specific glucuronide deconjugation (Kauffman, 1987; Sperker et al., 1997; De Graaf et al., 2002) should be considered as one of the most interesting hypothesis, especially when supported by numerous fundamental studies performed on various flavonoid glucuronides (O'Leary et al., 2001; Bartholomé et al., 2010; Lu et al., 2014).

\section{Activity of sulfate metabolites}

Recent HT bioavailability data demonstrated that sulfated metabolites appear to be the most abundant among the HT phase II metabolites (González-Santiago et al., 2010b; Kotronoulas et al., 2013; Pérez-Mañá et al., 2015b), thus drawing attention to sulfoconjugated forms. Interestingly, a recent report evaluating the antioxidant effect of $\mathrm{HT}$ and Tyr sulfate metabolites in intestinal cells 
(Caco-2) found that HT and Tyr sulfates displayed an efficiency in protecting cells comparable to that of the parent compounds (Atzeri et al., 2016).

\section{Beyond direct antioxidant mechanisms}

Along with the modulation of oxidative status, the non-antioxidant activities of metabolites are also of great interest since such events have been reported in several dietary intervention studies (Marrugat et al., 2004; Covas et al., 2006b). Among them, impact on gene expression should be considered as it has been shown to be dose-dependently modulated by phenolic compounds (Konstantinidou et al., 2013). In a recent report, the effect of the 3-O- and 4-O-glucuronides of HT on the prevention of endoplasmic reticulum (ER) stress (a process mediated by the unfolded protein response and relevant for atherosclerosis development) has been examined in vitro (Giordano et al., 2015). Glucuronides were demonstrated to prevent chemically induced ER stress. It is noteworthy that the mechanisms involved in this effect do not match those involved for HT, thus stressing the concept that biological activities elicited by metabolites do not necessarily mimic those of the parent compound. Another recent study (Catalán et al., 2015) also examined a set of phase II HT metabolites, generated in the course of Caco-2 cell metabolism and purified as a complex fraction for their biological activity. The co-incubation of HT and the set of Caco-2 cell derived metabolites with human aortic endothelial cells (HAEC) indicate that both treatments significantly reduced the TNF- $\alpha$ induced secretion of cell adhesion molecules such as E-selectin, P-selectin, ICAM-1, and VCAM-1. However, only the HT metabolite fraction reduced further excretion of MCP-1 chemokine. On the basis of these results, the authors suggest that phase II metabolites, along with parent HT, might exert an endothelial dysfunction protection, a key factor in the pathogenesis of atherosclerosis, via modulation of cellular signaling. However, the mechanism of such action awaits further studies.

So far, only a few studies have been conducted to evaluate the bioactivities of phase II metabolites of $\mathrm{HT}$, and these were mainly focused on glucuronides or mixtures of Phase II metabolites. In vitro experiments with phase II metabolites (Deiana et al., 2011; Paiva-Martins et al., 
2013; Catalán et al., 2015; Giordano et al., 2015) suggest that they could be involved in some health promoting activities via mechanisms of action that differ from the parent compound. In addition to glucuronides, other major phase II metabolites of HT (among them recently acknowledged HT sulfates and specifically 3'-O-sulfate (González-Santiago et al., 2010a; Kotronoulas et al., 2013; Atzeri et al., 2016)), phase II metabolites of its methylated conjugates (Turner et al., 2005) and oxidized metabolites (D'Angelo et al., 2001; González-Santiago et al., 2010b), as well as reduced and glucuronoconjugated forms of aglycones (Pinto et al., 2011), should be also tested for their biological activities.

\section{Analysis of hydroxytyrosol, tyrosol and their metabolites in biological matrices}

The selection of the appropriate method of quantifying the concentration of HT, Tyr and their metabolites is critical to obtain reliable information about their bioavailability and metabolism. As already explained, these phenolic compounds undergo extensive metabolism and, consequently, they are found in biological samples at very low concentrations.

\section{Total hydroxytyrosol and tyrosol analysis (hydrolytic treatments)}

HT and Tyr are recognized and handled by the human body as xenobiotic compounds. As a consequence, after (and during) their absorption, they undergo extensive first-pass and phase $\mathrm{I} / \mathrm{II}$ metabolism that results in considerable conjugation ( $>90 \%$ ) (Miró-Casas et al., 2003a). For this reason, measuring only the free forms of $\mathrm{HT}$ and Tyr is not representative of their intake and metabolism.

Following this observation, some authors adopted the strategy of hydrolyzing the conjugated metabolites in order to obtain an indirect measurement of them. An initial option taken was the enzymatic hydrolysis with $\beta$-glucuronidase, used in rat urine (Visioli et al., 2001; Del Boccio et al., 2003) and rat plasma (Del Boccio et al., 2003; Bazoti et al., 2010). Another possibility is the use of a chemical (acidic) hydrolysis of the samples. This has been widely employed in human urine (Visioli et 
al., 2000; Miró-Casas et al., 2001a; Miró-Casas et al., 2001b) and rat plasma (Bai et al., 1998; RuizGutierrez et al., 2000; Rodríguez-Gutiérrez et al., 2011). Following both hydrolytic procedures, an indirect estimation of $\mathrm{HT}$ and Tyr conjugated forms was made possible by measuring the released free forms of HT and Tyr. In the previous studies, the results were generally given as total amounts of HT and Tyr. It is worth mentioning the study in which the same plasma and urine samples were hydrolyzed using chemical or enzymatic hydrolysis, enabling a comparison between both hydrolytic methods. In both plasma and urine, enzymatic hydrolysis was able to identify only around $65 \%$ of the HT detected using acidic hydrolysis (Miró-Casas et al., 2003a). No data have been published concerning the use of combined hydrolytic methods. The difference between the results obtained with both methods lies on the fact that $\beta$-glucuronidase mainly quantitatively hydrolyzes the glucuronide conjugates. The sulfatase activity of the enzyme preparation is too weak to hydrolyze quantitatively sulfate conjugates whereas chemical hydrolysis allows the determination of other conjugated metabolites (e.g. sulfates or conjugated with glutathione).

Consequently, the resulting concentrations measured in these studies was a better approach than measuring only the free forms although it was not representative of all the HT and Tyr metabolites. Indeed, for none of the previous strategies was hydrolysis complete, and in both cases it was mainly limited to the measurement of glucuronides and free forms, leaving the other forms practically unbroken. For this reason, the real total content of HT and Tyr was underestimated. In addition, hydrolysis did not account for the principal $O$-methylated metabolites of $\mathrm{HT}$ and Tyr such as HVAlc or HVA. Some authors solved this last underestimation by measuring HVAlc (Miró-Casas et al., 2003a), whereas others included the analysis of HT-acetate, HVAlc, and HVA (Bazoti et al., 2010).

\section{Analysis of hydroxytyrosol, tyrosol and their main metabolites (non-hydrolytic treatments)}

Since the methods including hydrolysis failed to provide further information about the metabolism of HT and Tyr, another strategy was taken. The following step was the measurement of the concentrations of free and conjugated forms of $\mathrm{HT}$ and Tyr in biological samples as far as 
reference substances were available. This information may be relevant since, as already explained, it has been reported that the metabolites can exert a beneficial effect (Tuck et al., 2002; Atzeri et al., 2016) and the profile may be dose-dependent (Kotronoulas et al., 2013). The new approach presented an analytical challenge due to (1) the low concentrations of the different metabolites in biological matrices and (2) the wide array of compounds with varying polarities and chemical behaviors. Hence, an analytical procedure may be appropriate for the extraction and recovery of one compound but detrimental for another.

In 2006, the first direct (non-hydrolytic) method to analyze HT metabolites was described in human LDL samples (de la Torre-Carbot et al., 2006). The metabolites measured were HT monoglucuronide and monosulfate, Tyr glucuronide and sulfate, and HVA sulfate. In 2009, the group of Suárez et al. analyzed sulfates and glucuronides of Tyr and HT in human plasma samples (Suárez et al., 2009). In 2010, Khymenets et al. used a similar method in human urine samples including the analysis of HT and Tyr glucuronides. Subsequent studies reported an analysis of human plasma after phenol-enriched olive oil quantifying the free forms, HT sulfate, HT acetate sulfate, HVA, and HVA sulfate (Rubió et al., 2012). Finally, in 2013, Kotronoulas et al. reported an analytical method for the determination of HT, Tyr and metabolites in rat urine. In this study, a total of 9 compounds were monitored and thioether adducts of HT with glutathione were reported for the first time (Kotronoulas et al., 2013).

One of the main drawbacks in these analytical methods was the lack of appropriate standards of the metabolites, which hindered the quantification of these compounds. Several research groups used calibration curves of the free forms to quantify the concentrations of the metabolites (de la Torre-Carbot et al., 2006; Suárez et al., 2009; Rubió et al., 2012). Due to the different polarity and chemical behavior among the free forms and conjugates, the former may not be extracted and eluted at the same rate as the latter. The quantification of HT metabolites using non appropriate calibration curves might increase the risk of systematic inaccuracies in the obtained 
results. Another issue that hampers the proper quantification of HT and its metabolites is the use of internal standards with a chemical structure that markedly differs from the analyte of interest.

In order to overcome these challenges, Khymenets et al., firstly synthesized standards of HT and Tyr glucuronides. Secondly, the authors designed and synthesized a custom-made internal standard containing a glucuronic acid moiety in its structure [3-(4'-Hydroxyphenyl)propanol 4'-O- $\beta$ D-glucuronide] (Khymenets et al., 2006). The use of this strategy provided a reliable quantification of HT and Tyr glucuronides (Khymenets et al., 2010). Recent studies followed the previous approach and included additional metabolites (e.g. sulfates and $\mathrm{N}$-acetylcysteine derivatives) (Kotronoulas et al., 2013; Pérez-Mañá et al., 2015b).

\section{Extraction procedures}

A crucial part of the method is the extraction of the phenols from the biological matrix following hydrolysis. Most of the described methods performed either a liquid-liquid extraction (LLE) (Miró-Casas et al., 2001b; Del Boccio et al., 2003) or a solid-phase extraction (SPE) (Ruiz-Gutierrez et al., 2000; Miró-Casas et al., 2001a; de la Torre-Carbot et al., 2006; Bazoti et al., 2010; Khymenets et al., 2011; Rodríguez-Gutiérrez et al., 2011; Orozco-Solano et al., 2012; Domínguez-Perles et al., 2015). The SPE procedure has become more usual nowadays due to the fact that it provides cleaner extracts, and reduces the matrix effect, at the same time it increases the selectivity and reproducibility of the analysis.

Suárez et al. introduced the use of microelution plates in human plasma samples. The authors compared an SPE method with the microelution plate one. In the SPE, there was a higher suppression of the signal due to an increased matrix effect. The use of microelution plates was faster and allowed for a lower sample volume load. (Suárez et al., 2009). 


\section{Instrumentation}

The first developed analytical methods aiming to detect and measure HT concentrations in biological matrices used high-performance liquid chromatography with ultraviolet detection (HPLCUV) (Ruiz-Gutierrez et al., 2000; D'Angelo et al., 2001). The low molecular weights of HT and Tyr (154 and $138 \mathrm{~g} / \mathrm{mol}$, respectively), and the presence of hydroxyl groups in their chemical structures, allows these compounds to be derivatized and subsequently analyzed by gas chromatography coupled to mass spectrometry (GC/MS). In comparison with HPLC-UV, this technique has the advantage of presenting a higher selectivity and sensitivity. Consequently, GC/MS has been widely used in the analysis of HT and Tyr (Bai et al., 1998; Visioli et al., 2000; Miró-Casas et al., 2001b; Visioli et al., 2001). The use of liquid chromatography coupled to mass spectrometry (LC/MS) offers the possibility of accurately quantifying the levels of HT and Tyr. Finally, LC coupled to triple quadrupole mass spectrometers (LC-MS/MS) results in an increase in sensitivity and allows the direct measurement of phase II HT and Tyr metabolites (e.g. glucuronides, sulfates), thus overcoming the above-mentioned partial recoveries of the hydrolytic procedures (Del Boccio et al., 2003; de la TorreCarbot et al., 2006; Khymenets et al., 2011; Orozco-Solano et al., 2012; Kotronoulas et al., 2013; Serra et al., 2013; Domínguez-Perles et al., 2015; Pérez-Mañá et al., 2015b).

\section{Analysis of free hydroxytyrosol in human plasma}

Most of the previously mentioned methods, although suitable to determine the plasma or urinary concentrations of HT (and also Tyr) metabolites in humans, do not allow the measuring of low amounts of free HT that remain unaltered in human blood following the intake of olive oil. The metabolism that this phenolic compound undergoes is so extensive that the concentrations of free HT in body fluids were considered almost undetectable until recently (De la Torre, 2008).

HT plasma levels have been measured in some studies in which pure HT was administered to rats (Ruiz-Gutierrez et al., 2000) and humans (González-Santiago et al., 2010a). However, the nutraceutical doses of HT administered in these studies were significantly higher than dietetic ones. 
The sensitivity of the methods employed, although appropriate to detect free HT in plasma after non-dietetic doses, does not allow the identification of the low concentrations of free HT that follow olive oil or red wine intake.

The free (non-conjugated) fraction of HT and Tyr was previously reported in human urine following olive oil intake (Miró-Casas et al., 2001b; Weinbrenner et al., 2004). The percentages of HT and Tyr free forms represented 6 and 14\%, respectively, of the amounts recovered after hydrolytic treatment. However, the real percentages of the free forms might be even lower considering that hydrolysis did not release all the conjugates. Subsequent studies by the same authors concluded that, in the case of plasma, the percentage of free $\mathrm{HT}$ is less than $2 \%$, compared to the conjugated forms. In fact, they were not able to detect the free forms of Tyr and HT (Miró-Casas et al., 2003a).

Recently, the first direct method to measure free HT in human plasma has been reported (Pastor et al., 2016). The analytical method is based on the selective derivatization of the catechol group with benzylamine in the presence of an oxidant to form a fluorescent benzoxazole. Following this derivatization, $\mathrm{HT}$ is highly ionizable and stable and is detected by LC-MS/MS at a high sensitivity (LOD, $0.3 \mathrm{ng} / \mathrm{mL}$ ) and specificity. Once validated, the method was applied to the analysis of free HT in human plasma after olive oil intake. According to the authors (Pastor et al., 2016), the low amounts of free HT present in plasma after dietary doses $(0.3 \%$ of the dose administered) cannot explain a direct in vivo antioxidant activity of HT but could be the result of secondary mechanisms (e.g. transcriptomic effects or the activity of metabolites)(Konstantinidou et al., 2013; Catalán et al., 2015; Giordano et al., 2015).

\section{Concluding remarks}

During the last 20 years, the research on Tyr and HT has experienced an exponential increase due to the remarkable biological activities that these phenolic compounds display (PubMed search results by year: from 8 in 1995 to 130 in 2015). These include antioxidant, anti-inflammatory, cardioprotective, antitumor, antimicrobial, antidiabetic, and neuroprotective properties (Granados- 
Principal et al., 2010; Fernández-Mar et al., 2012; Rodríguez-Morató et al., 2015). Nowadays, it is well known that, after ingestion, both compounds are absorbed, metabolized into a wide multiplicity of phase I and II metabolites, distributed throughout the body, and excreted in urine (mainly in the form of sulfates and glucuronides). A great deal of progress has been made in relation to the analytical methods that detect and quantify Tyr, HT and their metabolites in biological matrices. Two key points have been crucial to achieve this progress: the use of LC-MS/MS, and the accessibility to pure standards of phase II metabolites. One of the examples that underscores the contribution of chemical synthesis and tandem mass spectrometry deals with sulfates. We now know that HT and Tyr sulfates may play a more important role than previously thought, as they are the predominant metabolites in plasma (Suárez et al., 2009) and urine (Pérez-Mañá et al., 2015b), and they exert biological actions by themselves (Atzeri et al., 2016).

The understanding of Tyr and HT complex metabolism has been hampered by several facts. These include (1) the endogenous generation of both phenolic compounds as products of tyramine and dopamine metabolism, respectively; (2) the increased endogenous generation of HT and Tyr by ethanol, (3) the ethanol-mediated increase in Tyr and HT absorption, and (4) the recently reported CYP-mediated endogenous conversion of Tyr to HT. This last observation opens the way to an interesting research field: the evaluation of health benefits associated with Tyr-containing foods (e.g. olive oil, wine) in the light of CYP genetic polymorphisms.

According to the results from HT pharmacokinetic studies following the intake of virgin olive oil, the amounts of the unaltered drug that reach plasma are too low to exert in vivo direct antioxidant activities. Therefore the strong antioxidant activities reported in vitro are of limited relevance in vivo. The discrepancy between the in vitro and in vivo results highlights the importance that the concentrations tested in vitro should include the conjugated forms (besides free forms) and be compatible with those attainable in vivo after the intake of realistic doses of HT- and Tyrcontaining foods. Indeed, most of the reported activities of HT and Tyr are focused on the free forms 
whereas only a few recent reports have evaluated the activity of the metabolites. Consequently, it is to be hoped that future studies on HT and Tyr biological activities will give more weight to the phase I/II metabolites.

Despite the recent advances in HT and Tyr metabolisms, the specific mechanism of action of these compounds deserves further study. According to the present data, it seems that the conjugated forms display biological activities related to the free forms, but probably through different mechanisms of actions. The possibility of deconjugation represents an additional challenge when trying to credit a particular biological effect to a metabolite. Besides the typical direct antioxidant capacity, phytochemicals are thought to contribute to beneficial effects through mechanisms independent of such capacity (e.g. by interaction with nuclear receptors or by stimulating cell signaling pathways that result in increased expression of genes) (Virgili and Marino, 2008).

Another interesting field that remains to be explored in more detail is the assessment of the microbial metabolism of $\mathrm{HT}$ and Tyr. During the last decade, the number of original articles related to gut microbiota has grown exponentially. For the moment, a couple of in vitro studies (Corona et al., 2006; Mosele et al., 2014) and one study in rats (Lin et al., 2013) have evaluated the microbiotamediated metabolism of olive oil phenolic compounds. Additional studies are needed to elucidate the bacterial metabolites of these compounds and their corresponding impact on biological activities.

Finally, according to toxicity studies, HT is considered a safe compound. When it was tested at concentrations that exceed those attainable after dietary intake, it was found to be non-genotoxic and non-mutagenic (Auñon-Calles et al., 2013a; Auñon-Calles et al., 2013b). This lack of toxicity raises the possibility of using this compound as a nutraceutical, as a strategy to administer higher doses than those attainable with diet. In fact, functional olive oils (enriched with their own phenolic compounds) (Farràs et al., 2015; Valls et al., 2015) and phenolic-rich olive leaf extracts (De Bock et al., 2013b; García-Villalba et al., 2014; Lockyer et al., 2015) have been administered in clinical trials. 
Moreover, pure HT has also been administered in a couple of human studies (González-Santiago et al., 2010a; Crespo et al., 2015). It is expected that future scientific reports in general, and clinical trials in particular, will allow the determining of the extent to which the promising profile of Tyr and HT can be translated into in vivo beneficial health effects.

\section{Acknowledgements}

The authors would like to thank Dr. Klaus Langohr for his assistance with statistical analyses.

\section{Declaration of interest statement}

This work was supported by grants from Instituto de Salud Carlos III FEDER, (PI14/00072) and from DIUE de la Generalitat de Catalunya (2014SGR 680). JRM was supported by a FI-DGR2012 predoctoral fellowship from the Generalitat de Catalunya and CPM was supported by a Juan Rodés fellowship (ISCIII, JR, 15/00005). CIBER de Fisiopatología de la Obesidad y Nutrición (CIBEROBN) is an initiative of the Instituto de Salud Carlos III, Madrid, Spain.

The authors report no declarations of interest.

\section{References}




\section{References}

Almeida C, Duarte IF, Barros A, Rodrigues J, Spraul M et al. (2006) Composition of Beer by 1H NMR Spectroscopy: Effects of Brewing Site and Date of Production. J Agr Food Chem, 54, 700-06.

Atzeri A, Lucas R, Incani A, Penalver P, Zafra-Gomez A et al. (2016) Hydroxytyrosol and tyrosol sulfate metabolites protect against the oxidized cholesterol pro-oxidant effect in Caco-2 human enterocyte-like cells. Food Funct.

Auñon-Calles D, Canut L, Visioli F (2013a) Toxicological evaluation of pure hydroxytyrosol. Food Chem Toxicol, 55, 498-504.

Auñon-Calles D, Giordano E, Bohnenberger S, Visioli F (2013b) Hydroxytyrosol is not genotoxic in vitro. Pharmacol Res, 74, 87-93.

Bai C, Yan X, Takenaka M, Sekiya K, Nagata T (1998) Determination of Synthetic Hydroxytyrosol in Rat Plasma by GC-MS. J Agr Food Chem, 46, 3998-4001.

Bartholomé R, Haenen G, Hollman PCH, Bast A, Dagnelie PC et al. (2010) Deconjugation Kinetics of Glucuronidated Phase II Flavonoid Metabolites by beta-glucuronidase from Neutrophils. Drug Metab Pharmacok, 25, 379-87.

Bayram B, Ozcelik B, Schultheiss G, Frank J, Rimbach G (2013) A validated method for the determination of selected phenolics in olive oil using high-performance liquid chromatography with coulometric electrochemical detection and a fused-core column. Food Chem, 138, 1663-69.

Bazoti FN, Gikas E, Tsarbopoulos A (2010) Simultaneous quantification of oleuropein and its metabolites in rat plasma by liquid chromatography electrospray ionization tandem mass spectrometry. Biomed Chromatogr, 24, 506-15.

Bendini A, Cerretani L, Carrasco-Pancorbo A, Gómez-Caravaca A, Segura-Carretero A et al. (2007) Phenolic Molecules in Virgin Olive Oils: a Survey of Their Sensory Properties, Health Effects, Antioxidant Activity and Analytical Methods. An Overview of the Last Decade Molecules, 12, 1679. 
Bernini R, Merendino N, Romani A, Velotti F (2013) Naturally Occurring Hydroxytyrosol: Synthesis and Anticancer Potential. Curr Med Chem, 20, 655-70.

Bogani P, Galli C, Villa M, Visioli F (2007) Postprandial anti-inflammatory and antioxidant effects of extra virgin olive oil. Atherosclerosis, 190, 181-86.

Bordiga M, Lorenzo C, Pardo F, Salinas MR, Travaglia F et al. (2016) Factors influencing the formation of histaminol, hydroxytyrosol, tyrosol, and tryptophol in wine: Temperature, alcoholic degree, and amino acids concentration. Food Chem, 197, Part B, 1038-45.

Briante R, Febbraio F, Nucci R (2003) Antioxidant Properties of Low Molecular Weight Phenols Present in the Mediterranean Diet. J Agr Food Chem, 51, 6975-81.

Bulotta S, Celano M, Lepore SM, Montalcini T, Pujia A et al. (2014) Beneficial effects of the olive oil phenolic components oleuropein and hydroxytyrosol: focus on protection against cardiovascular and metabolic diseases. J Trans/ Med, 12, 219-19.

Campesi I, Romani A, Marino M, Franconi F (2014) Phenolic Compounds from a Sex-Gender Perspective, in: Recent Advances in Polyphenol Research, pp 327-39, John Wiley \& Sons, Ltd.

Carrasco-Pancorbo A, Cerretani L, Bendini A, Segura-Carretero A, Del Carlo M et al. (2005) Evaluation of the Antioxidant Capacity of Individual Phenolic Compounds in Virgin Olive Oil. J Agr Food Chem, 53, 8918-25.

Caruso D, Visioli F, Patelli R, Galli C, Galli G (2001) Urinary excretion of olive oil phenols and their metabolites in humans. Metabolism, 50, 1426-28.

Catalán Ú, López de las Hazas M-C, Rubió L, Fernández-Castillejo S, Pedret A et al. (2015) Protective effect of hydroxytyrosol and its predominant plasmatic human metabolites against endothelial dysfunction in human aortic endothelial cells. Mol Nutr Food Res, 59, 2523-36.

Corona G, Tzounis X, Assunta Dessì M, Deiana M, Debnam ES et al. (2006) The fate of olive oil polyphenols in the gastrointestinal tract: Implications of gastric and colonic microfloradependent biotransformation. Free Radical Res, 40, 647-58. 
Covas M-I, de la Torre K, Farré-Albaladejo M, Kaikkonen J, Fitó M et al. (2006a) Postprandial LDL phenolic content and LDL oxidation are modulated by olive oil phenolic compounds in humans. Free Radical Bio Med, 40, 608-16.

Covas M-I, Nyyssönen K, Poulsen HE, Kaikkonen J, Zunft H-JF et al. (2006b) The Effect of Polyphenols in Olive Oil on Heart Disease Risk Factors: A Randomized Trial. Ann Intern Med, 145, 333-41.

Covas M-I, Ruiz-Gutiérrez V, de la Torre R, Kafatos A, Lamuela-Raventós RM et al. (2006c) Minor Components of Olive Oil: Evidence to Date of Health Benefits in Humans. Nutr Rev, 64, S20S30.

Covas MI, de la Torre R, Fitó M (2015) Virgin olive oil: a key food for cardiovascular risk protection. $\mathrm{Br}$ J Nutr, 113 Suppl 2, S19-28.

Covas MI, Miro-Casas E, Fito M, Farre-Albadalejo M, Gimeno E et al. (2003) Bioavailability of tyrosol, an antioxidant phenolic compound present in wine and olive oil, in humans. Drugs Exp Clin Res, 29, 203-6.

Crespo MC, Tomé-Carneiro J, Burgos-Ramos E, Loria Kohen V, Espinosa MI et al. (2015) One-week administration of hydroxytyrosol to humans does not activate Phase II enzymes. Pharmacol Res, 95-96, 132-37.

Chen W, Becker T, Qian F, Ring J (2014) Beer and beer compounds: physiological effects on skin health. J Eur Acad Dermatol, 28, 142-50.

Cheng Z, Ren J, Li Y, Chang W, Chen Z (2002) Study on the multiple mechanisms underlying the reaction between hydroxyl radical and phenolic compounds by qualitative structure and activity relationship. Bioorgan Med Chem, 10, 4067-73.

Chiva-Blanch G, Arranz S, Lamuela-Raventos RM, Estruch R (2013) Effects of Wine, Alcohol and Polyphenols on Cardiovascular Disease Risk Factors: Evidences from Human Studies. Alcohol Alcoholism, 48, 270-77.

Chiva-Blanch G, Badimon L, Estruch R (2014) Latest Evidence of the Effects of the Mediterranean Diet in Prevention of Cardiovascular Disease. Curr Atheroscler Rep, 16, 1-7. 
D'Angelo S, Manna C, Migliardi V, Mazzoni O, Morrica P et al. (2001) Pharmacokinetics and metabolism of hydroxytyrosol, a natural antioxidant from olive oil. Drug Metab Dispos, 29, $1492-8$.

Davis VE, Brown H, Huff JA, Cashaw JL (1967) The alteration of serotonin metabolism to 5hydroxytryptophol by ethanol ingestion in man. J Lab Clin Med, 69, 132-40.

Davis VEWalsh MJ (1970) Alcohol, amines, and alkaloids: a possible biochemical basis for alcohol addiction. Science, 167, 1005-7.

Davis VE, Walsh MJ, Yamanaka Y (1970) Augmentation of alkaloid formation from dopamine by alcohol and acetaldehyde in vitro. J Pharmacol Exp Ther, 174, 401-12.

De Bock M, Derraik JG, Brennan CM, Biggs JB, Morgan PE et al. (2013a) Olive (Olea europaea L.) leaf polyphenols improve insulin sensitivity in middle-aged overweight men: a randomized, placebo-controlled, crossover trial. PLoS One, 8, e57622.

De Bock M, Thorstensen EB, Derraik JGB, Henderson HV, Hofman PL et al. (2013b) Human absorption and metabolism of oleuropein and hydroxytyrosol ingested as olive (Olea europaeaL.) leaf extract. Mol Nutr Food Res, 57, 2079-85.

De Graaf M, Boven E, Scheeren HW, Haisma HJ, Pinedo HM (2002) Beta-glucuronidase-mediated drug release. Curr Pharm Des, 8, 1391-403.

de la Torre-Carbot K, Jauregui O, Castellote Al, Lamuela-Raventos RM, Covas MI et al. (2006) Rapid high-performance liquid chromatography-electrospray ionization tandem mass spectrometry method for qualitative and quantitative analysis of virgin olive oil phenolic metabolites in human low-density lipoproteins. J Chromatogr A, 1116, 69-75.

De la Torre R (2008) Bioavailability of olive oil phenolic compounds in humans. Inflammopharmacology, 16, 245-7.

de la Torre R CM, Pujadas MA, Fitó M, Farré M (2006) Is dopamine behind the health benefits of red wine? Eur J Nutr, 45, 307-10. 
Deiana M, Incani A, Rosa A, Atzeri A, Loru D et al. (2011) Hydroxytyrosol glucuronides protect renal tubular epithelial cells against $\mathrm{H}_{2} \mathrm{O}_{2}$ induced oxidative damage. Chem-Biol Interact, 193, 23239.

Deiana M, Incani A, Rosa A, Corona G, Atzeri A et al. (2008) Protective effect of hydroxytyrosol and its metabolite homovanillic alcohol on $\mathrm{H} 2 \mathrm{O} 2$ induced lipid peroxidation in renal tubular epithelial cells. Food Chem Toxicol, 46, 2984-90.

Del Boccio P, Di Deo A, De Curtis A, Celli N, lacoviello L et al. (2003) Liquid chromatography-tandem mass spectrometry analysis of oleuropein and its metabolite hydroxytyrosol in rat plasma and urine after oral administration. J Chromatogr B 785, 47-56.

Di Tommaso D, Calabrese R, Rotilio D (1998) Identification and Quantitation of Hydroxytyrosol in Italian Wines. J High Res Chromatogr, 21, 549-53.

Domínguez-Perles R, Auñón D, Ferreres F, Gil-Izquierdo A (2015) Gender differences in plasma and urine metabolites from Sprague-Dawley rats after oral administration of normal and high doses of hydroxytyrosol, hydroxytyrosol acetate, and DOPAC. Eur J Nutr, 1-10.

Dragoni S, Gee J, Bennett R, Valoti M, Sgaragli G (2006) Red wine alcohol promotes quercetin absorption and directs its metabolism towards isorhamnetin and tamarixetin in rat intestine in vitro. Br J Pharmacol, 147, 765-71.

EFSA (2011) EFSA Panel on Dietetic Products, Nutrition and allergies (NDA) (2011) Scientific opinion on the substantiation of health claims related to polyphenols in olive oil and protection of LDL particles from oxidative damage. EFSA J 9, 2033. http://www.efsa.europa.eu/en/efsajournal/pub/2033. (accessed on August 2015).

Estruch R, Martínez-González MAn, Corella D, Salas-Salvadó J, Ruiz-Gutiérrez V et al. (2006) Effects of a Mediterranean-Style Diet on Cardiovascular Risk FactorsA Randomized Trial. Ann Intern Med, 145, 1-11.

Estruch R, Ros E, Salas-Salvadó J, Covas M-I, Corella D et al. (2013) Primary Prevention of Cardiovascular Disease with a Mediterranean Diet. New Eng J Med, 368, 1279-90. 
Farràs M, Castañer O, Martín-Peláez S, Hernáez Á, Schröder H et al. (2015) Complementary phenolenriched olive oil improves HDL characteristics in hypercholesterolemic subjects. A randomized, double-blind, crossover, controlled trial. The VOHF study. Mol Nutr Food Res, $59,1758-70$.

Fernández-Mar MI, Mateos R, García-Parrilla MC, Puertas B, Cantos-Villar E (2012) Bioactive compounds in wine: Resveratrol, hydroxytyrosol and melatonin: A review. Food Chem, 130, 797-813.

Fitó M, Cladellas M, de la Torre R, Martí J, Alcántara M et al. (2005) Antioxidant effect of virgin olive oil in patients with stable coronary heart disease: a randomized, crossover, controlled, clinical trial. Atherosclerosis, 181, 149-58.

Fitó M, Gimeno E, Covas MI, Miró E, López-Sabater Mdel C et al. (2002) Postprandial and short-term effects of dietary virgin olive oil on oxidant/antioxidant status. Lipids, 37, 245-51.

Forman HJ, Davies KJA, Ursini F (2014) How do nutritional antioxidants really work: Nucleophilic tone and para-hormesis versus free radical scavenging in vivo. Free Radical Bio Med, 66, 24-35.

García-Villalba R, Larrosa M, Possemiers S, Tomás-Barberán FA, Espín JC (2014) Bioavailability of phenolics from an oleuropein-rich olive (Olea europaea) leaf extract and its acute effect on plasma antioxidant status: comparison between pre- and postmenopausal women. Eur J Nutr, 53, 1015-27.

Gil-Izquierdo A, Vilaplana Peréz C, Auñon Calles D, García Flores LA (2014) Hydroxytyrosol and potential uses in cardiovascular diseases, cancer and AIDS. Front Nutr, 1.

Giordano E, Dangles O, Rakotomanomana N, Baracchini S, Visioli F (2015) 3-O-Hydroxytyrosol glucuronide and 4-0-hydroxytyrosol glucuronide reduce endoplasmic reticulum stress in vitro. Food Funct, 6, 3275-81.

Gomes VPM, Torres C, Rodríguez-Borges JE, Paiva-Martins F (2015) A Convenient Synthesis of Hydroxytyrosol Monosulfate Metabolites. J Agr Food Chem, 63, 9565-71. 
González-Santiago M, Fonollá J, Lopez-Huertas E (2010a) Human absorption of a supplement containing purified hydroxytyrosol, a natural antioxidant from olive oil, and evidence for its transient association with low-density lipoproteins. Pharmacol Res, 61, 364-70.

González-Santiago M, Fonollá J, Lopez-Huertas E (2010b) Human absorption of a supplement containing purified hydroxytyrosol, a natural antioxidant from olive oil, and evidence for its transient association with low-density lipoproteins. Pharmacological Research, 61, 364-70.

Granados-Principal S, Quiles JL, Ramirez-Tortosa CL, Sanchez-Rovira P, Ramirez-Tortosa MC (2010) Hydroxytyrosol: from laboratory investigations to future clinical trials. Nutr Rev, 68, 191-206.

Harman D (1956) Aging: A Theory Based on Free Radical and Radiation Chemistry. J Gerontol, 11, 298-300.

Hashimoto T, Ibi M, Matsuno K, Nakashima S, Tanigawa T et al. (2004) An endogenous metabolite of dopamine, 3,4-dihydroxyphenylethanol, acts as a unique cytoprotective agent against oxidative stress-induced injury. Free Radic Biol Med, 36, 555-64.

Holst BWilliamson G (2008) Nutrients and phytochemicals: from bioavailability to bioefficacy beyond antioxidants. Curr Opin Biotech, 19, 73-82.

Karkoula E, Skantzari A, Melliou E, Magiatis P (2012) Direct measurement of oleocanthal and oleacein levels in olive oil by quantitative (1)H NMR. Establishment of a new index for the characterization of extra virgin olive oils. J Agr Food Chem, 60, 11696-703.

Kauffman FC (1987) Conjugation-deconjugation reactions in drug metabolism and toxicity. Fed Proc, 46, 2434-45.

Khymenets O, Farré M, Pujadas M, Ortiz E, Joglar J et al. (2011) Direct analysis of glucuronidated metabolites of main olive oil phenols in human urine after dietary consumption of virgin olive oil. Food Chem, 126, 306-14.

Khymenets O, Fitó M, Touriño S, Muñoz-Aguayo D, Pujadas M et al. (2010) Antioxidant Activities of Hydroxytyrosol Main Metabolites Do Not Contribute to Beneficial Health Effects after Olive Oil Ingestion. Drug Metab Dispos, 38, 1417-21. 
Khymenets O, Joglar J, Clapés P, Parella T, Covas M-I et al. (2006) Biocatalyzed Synthesis and Structural Characterization of Monoglucuronides of Hydroxytyrosol, Tyrosol, Homovanillic Alcohol, and 3-(4'-Hydroxyphenyl)propanol. Adv Synth Cat, 348, 2155-62.

Konstantinidou V, Covas M-I, Sola R, Fitó M (2013) Up-to date knowledge on the in vivo transcriptomic effect of the Mediterranean diet in humans. Mol Nutr Food Res, 57, 772-83.

Konstantinidou V, Covas MI, Munoz-Aguayo D, Khymenets O, de la Torre R et al. (2010) In vivo nutrigenomic effects of virgin olive oil polyphenols within the frame of the Mediterranean diet: a randomized controlled trial. FASEB J, 24, 2546-57.

Kotronoulas A, Pizarro N, Serra A, Robledo P, Joglar J et al. (2013) Dose-dependent metabolic disposition of hydroxytyrosol and formation of mercapturates in rats. Pharmacol Res, 77, 4756.

Lin P, Qian W, Wang X, Cao L, Li S et al. (2013) The biotransformation of oleuropein in rats. Biomed Chromatogr, 27, 1162-67.

Lockyer S, Corona G, Yaqoob P, Spencer JPE, Rowland I (2015) Secoiridoids delivered as olive leaf extract induce acute improvements in human vascular function and reduction of an inflammatory cytokine: a randomised, double-blind, placebo-controlled, cross-over trial. Br J Nutr, 114, 75-83.

López de las Hazas M-C, Rubió L, Kotronoulas A, de la Torre R, Solà R et al. (2015) Dose effect on the uptake and accumulation of hydroxytyrosol and its metabolites in target tissues in rats. Molecular Nutrition \& Food Research, 59, 1395-99.

Lu Q-Y, Zhang L, Eibl G, Go VLW (2014) Overestimation of flavonoid aglycones as a result of the ex vivo deconjugation of glucuronides by the tissue $\beta$-glucuronidase. J Pharmaceut Biomed, 88 , 364-69.

Lucas R, Alcantara D, Morales JC (2009) A concise synthesis of glucuronide metabolites of urolithin-B, resveratrol, and hydroxytyrosol. Carbohyd Res, 344, 1340-46. 
Manna C, Galletti P, Maisto G, Cucciolla V, D'Angelo S et al. (2000) Transport mechanism and metabolism of olive oil hydroxytyrosol in Caco-2 cells. FEBS Letters, 470, 341-44.

Marrugat J, Covas M-I, Fitó M, Schröder H, Miró-Casas E et al. (2004) Effects of differing phenolic content in dietary olive oils on lipids and LDL oxidation. Eur J Nutr, 43, 140-47.

Miró-Casas E, Covas M-I, Farré M, Fitó M, Ortuño J et al. (2003a) Hydroxytyrosol Disposition in Humans. Clin Chem, 49, 945-52.

Miró-Casas E, Covas MI, Fitó M, Farré-Albadalejo M, Marrugat J et al. (2003b) Tyrosol and hydroxytyrosol are absorbed from moderate and sustained doses of virgin olive oil in humans. Eur J Clin Nutr, 57, 186-90.

Miró-Casas E, Farré Albadalejo M, Covas Planells MI, Fitó Colomer M, Lamuela Raventós RM et al. (2001a) Tyrosol Bioavailability in Humans after Ingestion of Virgin Olive Oil. Clin Chem, 47, $341-43$

Miró-Casas E, Farré Albaladejo M, Covas M-I, Rodriguez JO, Menoyo Colomer E et al. (2001b) Capillary Gas Chromatography-Mass Spectrometry Quantitative Determination of Hydroxytyrosol and Tyrosol in Human Urine after Olive Oil Intake. Anal Biochem, 294, 63-72.

Mosele Jl, Martín-Peláez S, Macià A, Farràs M, Valls R-M et al. (2014) Faecal microbial metabolism of olive oil phenolic compounds: In vitro and in vivo approaches. Mol Nutr Food Res, 58, 180919.

Nenadis N, Wang L-F, Tsimidou MZ, Zhang H-Y (2005) Radical Scavenging Potential of Phenolic Compounds Encountered in O. europaea Products as Indicated by Calculation of Bond Dissociation Enthalpy and Ionization Potential Values. J Agr Food Chem, 53, 295-99.

O'Leary KA, Day AJ, Needs PW, Sly WS, O'Brien NM et al. (2001) Flavonoid glucuronides are substrates for human liver $\beta$-glucuronidase. FEBS Letters, 503, 103-06.

Obied HK, Prenzler PD, Ryan D, Servili M, Taticchi A et al. (2008) Biosynthesis and biotransformations of phenol-conjugated oleosidic secoiridoids from Olea europaea L. Nat Prod Rep, 25, 116779. 
Orozco-Solano MI, Ferreiro-Vera C, Priego-Capote F, Luque de Castro MD (2012) Automated method for determination of olive oil phenols and metabolites in human plasma and application in intervention studies. J Chromatogr A, 1258, 108-16.

Ortega-García FPeragón J (2010) HPLC analysis of oleuropein, hydroxytyrosol, and tyrosol in stems and roots of Olea europaea L. cv. Picual during ripening. J Sci Food Agr, 90, 2295-300.

Paiva-Martins F, Silva A, Almeida V, Carvalheira M, Serra C et al. (2013) Protective Activity of Hydroxytyrosol Metabolites on Erythrocyte Oxidative-Induced Hemolysis. J Agr Food Chem, 61, 6636-42.

Panova NG, Veselovskaia NV, Medvedev AE (1997) Involvement of monoamine oxidases in formation of 4-hydroxyphenylethanol, major component of tribulin A. Vopr Med Khim, 43, 172-6.

Pastor A, Rodríguez-Morató J, Olesti E, Pujadas M, Pérez-Mañá C et al. (2016) Analysis of free hydroxytyrosol in human plasma following the administration of olive oil. J Chromatogr $A$, $1437,183-90$.

Pérez-Mañá C, Farre M, Pujadas M, Mustata C, Menoyo E et al. (2015a) Ethanol induces hydroxytyrosol formation in humans. Pharmacol Res, 95-96, 27-33.

Pérez-Mañá C, Farré M, Rodríguez-Morató J, Papaseit E, Pujadas M et al. (2015b) Moderate consumption of wine, through both its phenolic compounds and alcohol content, promotes hydroxytyrosol endogenous generation in humans. A randomized controlled trial. Mol Nutr Food Res, 59, 1213-16.

Pinto J, Paiva-Martins F, Corona G, Debnam ES, Jose Oruna-Concha M et al. (2011) Absorption and metabolism of olive oil secoiridoids in the small intestine. Br J Nutr, 105, 1607-18.

Piñeiro Z, Cantos-Villar E, Palma M, Puertas B (2011) Direct Liquid Chromatography Method for the Simultaneous Quantification of Hydroxytyrosol and Tyrosol in Red Wines. J Agr Food Chem, 59, 11683-89.

Rahman K (2007) Studies on free radicals, antioxidants, and co-factors. Clin Interv Aging, 2, 219-36. 
Roche M, Dufour C, Mora N, Dangles O (2005) Antioxidant activity of olive phenols: mechanistic investigation and characterization of oxidation products by mass spectrometry. Org Biomol Chem, 3, 423-30.

Rodríguez-Gutiérrez G, Wood S, Fernández-Bolaños Guzmán J, Duthie GG, de Roos B (2011) Determination of 3,4-dihydroxyphenylglycol, hydroxytyrosol and tyrosol purified from olive oil by-products with HPLC in animal plasma and tissues. Food Chem, 126, 1948-52.

Rodríguez-Morató J, Xicota L, Fitó M, Farré M, Dierssen M et al. (2015) Potential Role of Olive Oil Phenolic Compounds in the Prevention of Neurodegenerative Diseases. Molecules, 20, 465580.

Rubió L, Macià A, Valls RM, Pedret A, Romero M-P et al. (2012) A new hydroxytyrosol metabolite identified in human plasma: Hydroxytyrosol acetate sulphate. Food Chem, 134, 1132-36.

Rubió L, Serra A, Macià A, Piñol C, Romero M-P et al. (2014) In vivo distribution and deconjugation of hydroxytyrosol phase II metabolites in red blood cells: A potential new target for hydroxytyrosol. J Funct Foods, 10, 139-43.

Ruiz-Gutierrez V, Juan ME, Cert A, Planas JM (2000) Determination of hydroxytyrosol in plasma by HPLC. Anal Chem, 72, 4458-61.

Schröder H, de la Torre R, Estruch R, Corella D, Martínez-González MA et al. (2009) Alcohol consumption is associated with high concentrations of urinary hydroxytyrosol. Am J Clin Nutr, 90, 1329-35.

Serra A, Rubió L, Borràs X, Macià A, Romero M-P et al. (2012) Distribution of olive oil phenolic compounds in rat tissues after administration of a phenolic extract from olive cake. Mol Nutr Food Res, 56, 486-96.

Serra A, Rubió L, Macià A, Valls R-M, Catalán Ú et al. (2013) Application of dried spot cards as a rapid sample treatment method for determining hydroxytyrosol metabolites in human urine samples. Comparison with microelution solid-phase extraction. Anal Bioanal Chem, 405, 9179-92. 
Servili M, Sordini B, Esposto S, Urbani S, Veneziani G et al. (2014) Biological Activities of Phenolic Compounds of Extra Virgin Olive Oil. Antioxidants, 3, 1.

Sharp D (2003) Château resveratrol. Lancet, 361, 1404.

Sperker B, Backman JT, Kroemer HK (1997) The role of beta-glucuronidase in drug disposition and drug targeting in humans. Clin Pharmacokinet, 33, 18-31.

Suárez M, Romero MP, Macia A, Valls RM, Fernandez S et al. (2009) Improved method for identifying and quantifying olive oil phenolic compounds and their metabolites in human plasma by microelution solid-phase extraction plate and liquid chromatography-tandem mass spectrometry. J Chromatogr B 877, 4097-106.

Tacker M, Creaven PJ, Mclsaac WM (1970) Alteration in tyramine metabolism by ethanol. Biochem Pharmacol, 19, 604-7.

Tank AWWeiner H (1979) Ethanol-induced alteration of dopamine metabolism in rat liver. Biochem Pharmacol, 28, 3139-47.

Tripoli E, Giammanco M, Tabacchi G, Di Majo D, Giammanco S et al. (2005) The phenolic compounds of olive oil: structure, biological activity and beneficial effects on human health. Nutr Res Rev, $18,98-112$.

Tuck KL, Freeman MP, Hayball PJ, Stretch GL, Stupans I (2001) The In Vivo Fate of Hydroxytyrosol and Tyrosol, Antioxidant Phenolic Constituents of Olive Oil, after Intravenous and Oral Dosing of Labeled Compounds to Rats. J Nutr, 131, 1993-96.

Tuck KL, Hayball PJ, Stupans I (2002) Structural Characterization of the Metabolites of Hydroxytyrosol, the Principal Phenolic Component in Olive Oil, in Rats. J Agr Food Chem, 50, 2404-09.

Turner R, Etienne N, Alonso MG, de Pascual-Teresa S, Minihane AM et al. (2005) Antioxidant and anti-atherogenic activities of olive oil phenolics. Int J Vitam Nutr Res, 75, 61-70. 
Valls R-M, Farràs M, Suárez M, Fernández-Castillejo S, Fitó M et al. (2015) Effects of functional olive oil enriched with its own phenolic compounds on endothelial function in hypertensive patients. A randomised controlled trial. Food Chem, 167, 30-35.

Vasdev S, Gill V, Singal PK (2006) Beneficial effect of low ethanol intake on the cardiovascular system: possible biochemical mechanisms. Vasc Health Risk Manag, 2, 263-76.

Virgili FMarino M (2008) Regulation of cellular signals from nutritional molecules: a specific role for phytochemicals, beyond antioxidant activity. Free Radical Bio Med, 45, 1205-16.

Visioli F, Bellomo G, Galli C (1998) Free radical-scavenging properties of olive oil polyphenols. Biochem Biophys Res Commun, 247, 60-4.

Visioli FBernardini E (2011) Extra Virgin Olive Oils Polyphenols: Biological Activities. Curr Pharm Design, $17,786-804$.

Visioli F, Caruso D, Plasmati E, Patelli R, Mulinacci N et al. (2001) Hydroxytyrosol, as a component of olive mill waste water, is dose- dependently absorbed and increases the antioxidant capacity of rat plasma. Free Radic Res, 34, 301-5.

Visioli F, Galli C, Bornet F, Mattei A, Patelli R et al. (2000) Olive oil phenolics are dose-dependently absorbed in humans. FEBS Letters, 468, 159-60.

Visioli F, Galli C, Grande S, Colonnelli K, Patelli C et al. (2003) Hydroxytyrosol excretion differs between rats and humans and depends on the vehicle of administration. J Nutr, 133, 2612-5.

Visioli F, Poli A, Gall C (2002) Antioxidant and other biological activities of phenols from olives and olive oil. Med Res Rev, 22, 65-75.

Vissers MN, Zock PL, Katan MB (2004) Bioavailability and antioxidant effects of olive oil phenols in humans: a review. Eur J Clin Nutr, 58, 955-65.

Vissers MN, Zock PL, Roodenburg AJC, Leenen R, Katan MB (2002) Olive Oil Phenols Are Absorbed in Humans. J Nutr, 132, 409-17. 
Weinbrenner T, Fito M, Farre Albaladejo M, Saez GT, Rijken P et al. (2004) Bioavailability of phenolic compounds from olive oil and oxidative/antioxidant status at postprandial state in healthy humans. Drugs Exp Clin Res, 30, 207-12.

Witztum JL (1994) The oxidation hypothesis of atherosclerosis. Lancet, 344, 793-95. 


1
2
3
4
5
6
7
8
9
10
11
12
13
14
15
16
17
18
19
20
21
22
23
24
25
26
27
28
29
30
31
32
33
34
35
36
37
38
39
40
41
42
43
44
45
46
47
48
49
50
51
52
53
54
55
56
57
58
60

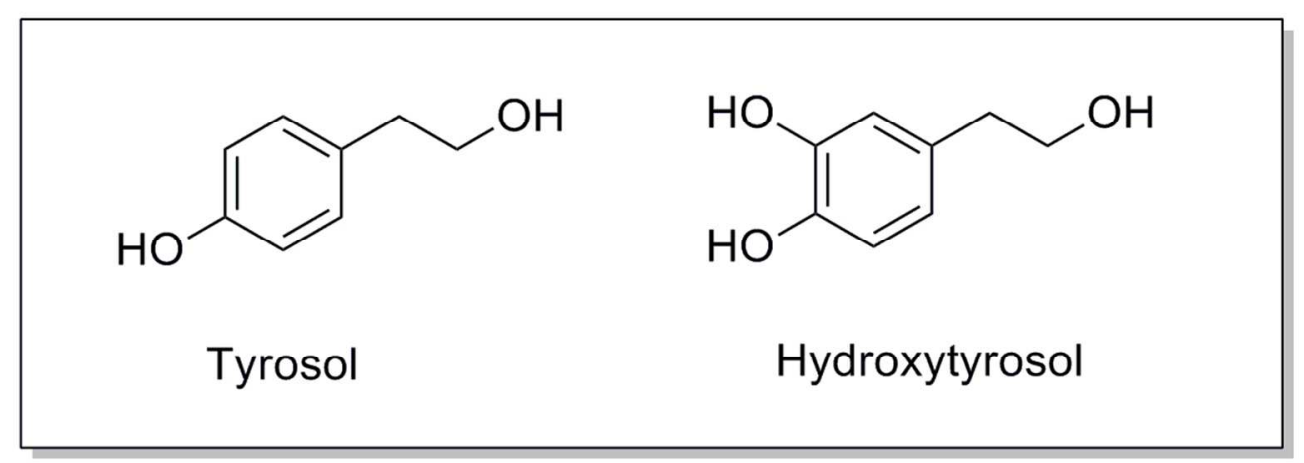

Figure 1. Chemical structures of tyrosol and hydroxytyrosol. $98 \times 35 \mathrm{~mm}(300 \times 300 \mathrm{DPI})$ 


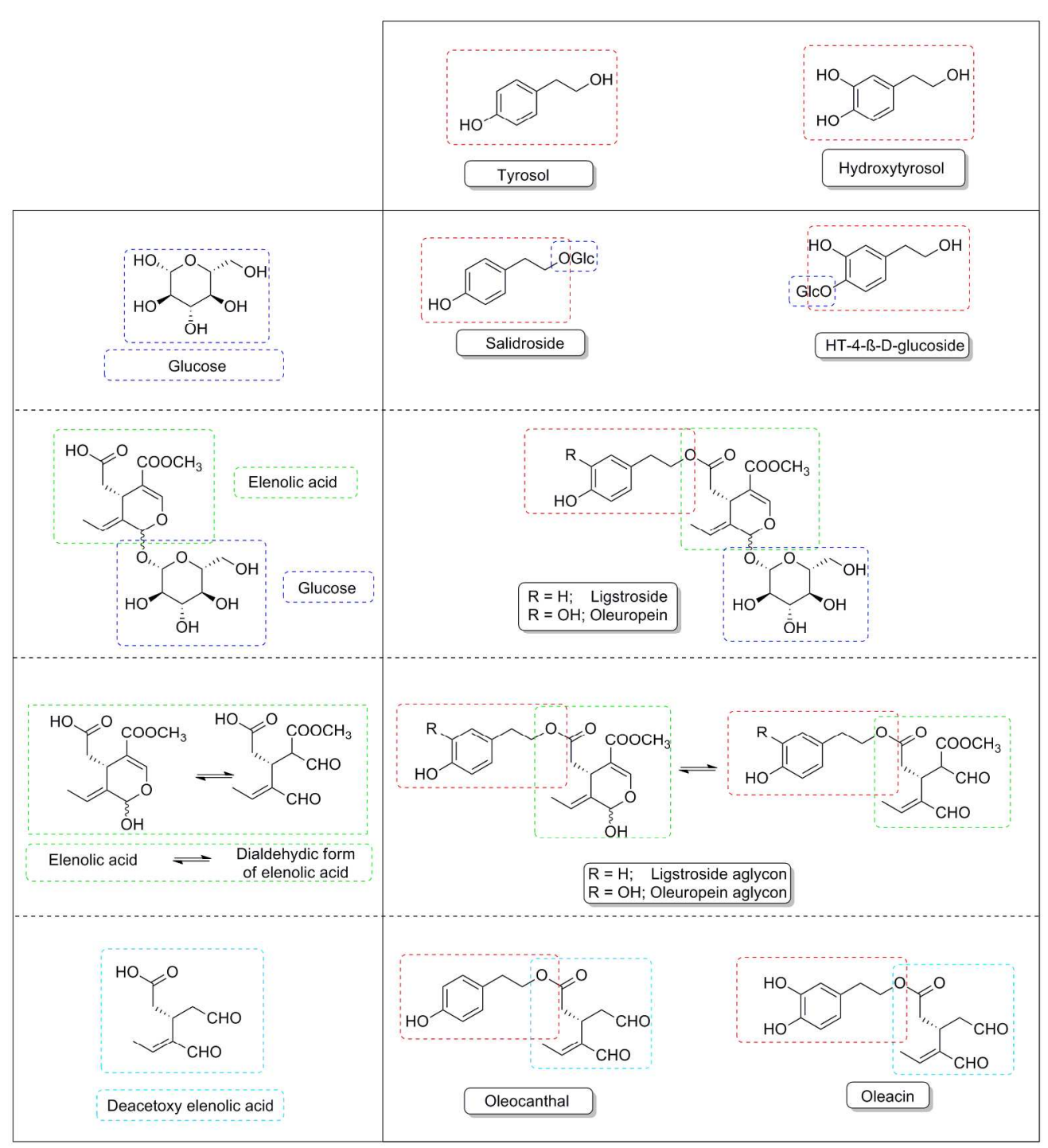

Figure 2. Glycosides and esters of tyrosol and hydroxytyrosol present in olive fruits and olive oil. $229 \times 249 \mathrm{~mm}(300 \times 300$ DPI) 


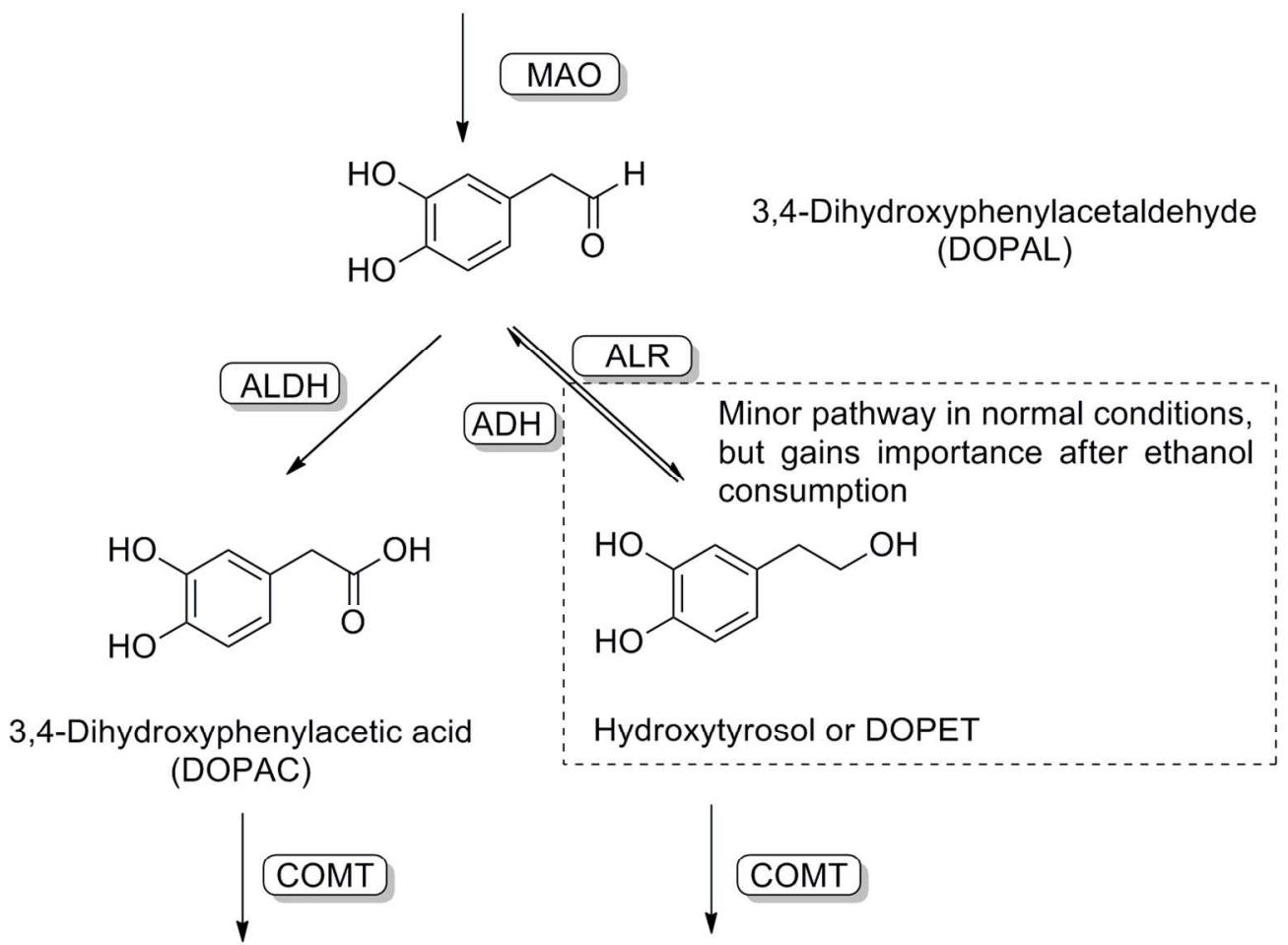<smiles>COc1cc(CC(=O)O)ccc1O</smiles>

Homovanillic acid

(HVA)

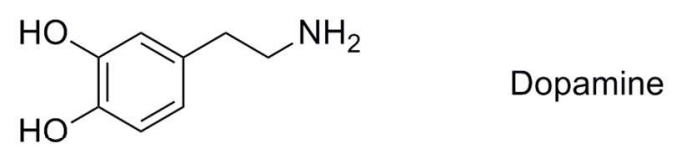
(DOPAL)<smiles>COc1cc(CCO)ccc1O</smiles>

\section{Homovanillyl alcohol} (HVAlc or MOPET)

Figure 3. Dopamine oxidative metabolism diagram. Abbreviations: ALR: Aldehyde/aldose reductase; ADH: Alcohol dehydrogenase; ALDH: Aldehyde dehydrogenase; MAO: Monoamine oxidase. $134 \times 146 \mathrm{~mm}(300 \times 300 \mathrm{DPI})$ 


\section{Tyramine}<smiles>COc1ccccc1</smiles>

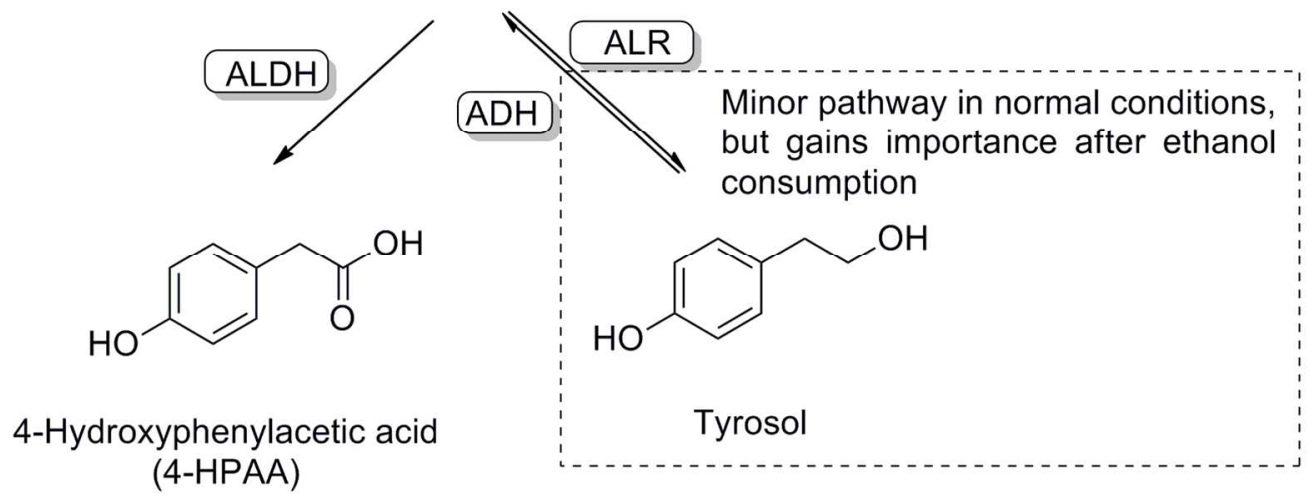

Figure 4. Tyramine oxidative metabolism diagram. Abbreviations: ALR: Aldehyde/aldose reductase; ADH: Alcohol dehydrogenase; ALDH: Aldehyde dehydrogenase; MAO: Monoamine oxidase. $131 \times 99 \mathrm{~mm}(300 \times 300 \mathrm{DPI})$ 

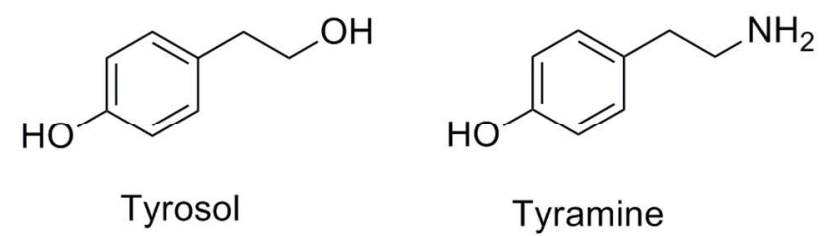

Figure 5. Chemical structures of tyrosine, tyrosol, and tyramine. $125 \times 23 \mathrm{~mm}(300 \times 300 \mathrm{DPI})$ 
Figure 6. Schematic representation of the postulated effects of ethanol on tyrosol and hydroxytyrosol production. Blue arrows indicate an increase whereas the red arrow indicates an inhibition.

$219 \times 136 \mathrm{~mm}(96 \times 96 \mathrm{DPI})$ 


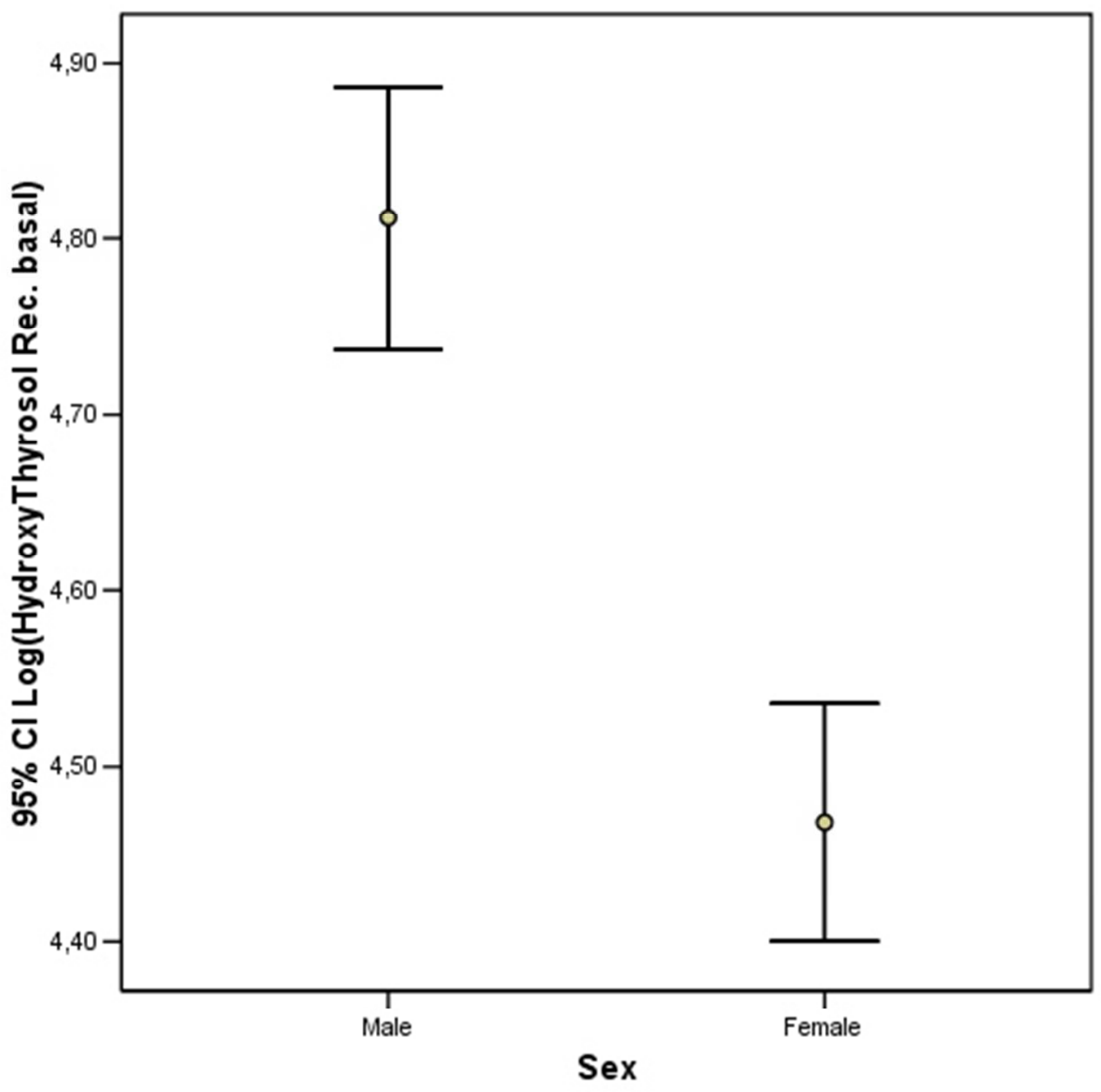

Figure 7. Analysis of covariance of HT urinary concentrations adjusting for ethyl glucuronide concentrations and gender. $132 \times 132 \mathrm{~mm}(96 \times 96 \mathrm{DPI})$ 
1

2

3

4

5

6

7

8

9

10

11

12

13

14

15

16

17

18

19

20

21

22

23

24

25

26

27

28

29

30

31

32

33

34

35

36

37

38

39

40

41

42

43

44

45

46

47

48

49

50

51

52

53

54

55

56

57

58

59

60

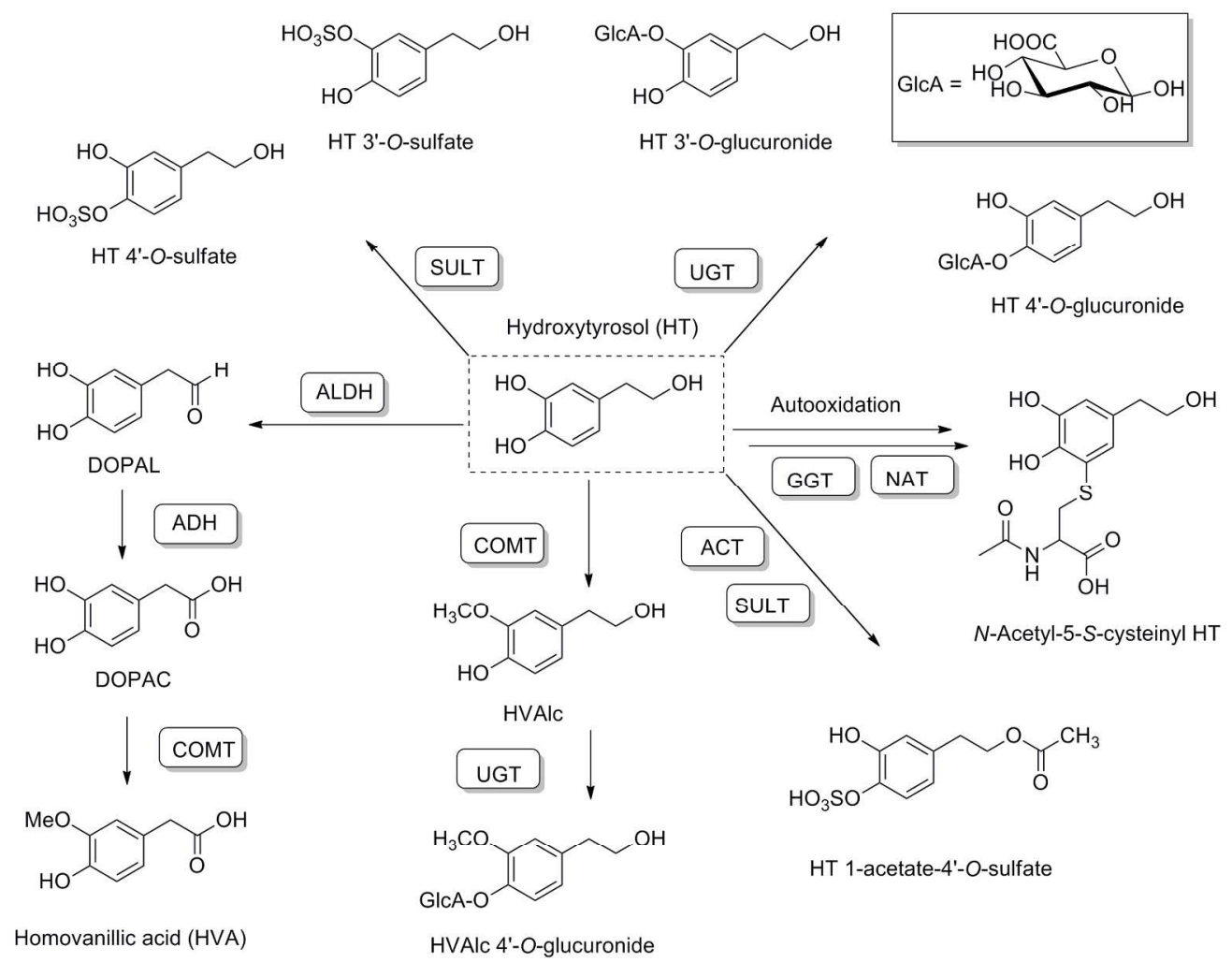

Figure 8. Hydroxytyrosol (HT) metabolic pathways. Abbreviations: ACT: O-Acetyl transferase; ADH: Alcohol dehydrogenase; ALDH: Aldehyde dehydrogenase; COMT: Catechol-O-methyl transferase; GGT: $\gamma$-Glutamyl transferase; GlcA: Glucuronic acid; HVAlc: Homovanillyl alcohol; NAT: $N$-acetyl transferase; UGT: UDPglucuronosyl transferase; SULT: Sulfotransferase. $198 \times 155 \mathrm{~mm}(300 \times 300 \mathrm{DPI})$ 